\title{
Ontology-Based Analysis of Manufacturing Processes: Lessons Learned from the Case Study of Wire Harness Production
}

\author{
László Nagy, Tamás Ruppert $\mathbb{D}$, and János Abonyi \\ MTA-PE Lendület Complex Systems Monitoring Research Group, Department of Process Engineering, University of Pannonia, \\ Egyetem u. 10, POB 158, Veszprém H-8200, Hungary
}

Correspondence should be addressed to Tamás Ruppert; ruppert@abonyilab.com

Received 7 June 2021; Revised 13 October 2021; Accepted 25 October 2021; Published 19 November 2021

Academic Editor: Muhammad Javaid

Copyright @ 2021 László Nagy et al. This is an open access article distributed under the Creative Commons Attribution License, which permits unrestricted use, distribution, and reproduction in any medium, provided the original work is properly cited.

Effective information management is critical for the development of manufacturing processes. This paper aims to provide an overview of ontologies that can be utilized in building Industry 4.0 applications. The main contributions of the work are that it highlights ontologies that are suitable for manufacturing management and recommends the multilayer-network-based interpretation and analysis of ontology-based databases. This article not only serves as a reference for engineers and researchers on ontologies but also presents a reproducible industrial case study that describes the ontology-based model of a wire harness assembly manufacturing process.

\section{Introduction}

Managing information and data from production systems is critical for digital transformation, especially in Industry 4.0 applications, where the horizontal and vertical integration of systems requires more efficient data processing. More efforts have been made to standardize this area, such as the ANSI/ ISA-95 international standard or RAMI 4.0 (Reference Architectural Model Industrie 4.0) [1]. Furthermore, there are ongoing studies in the field of different methodologies and data structurization that aim to support productionrelated decision-making processes [2] or create models without simulation software-specific knowledge [3]. For a similar purpose, process mining solutions were also developed to discover, analyze, and improve business processes based on event logs of information systems [4, 5].

Semantic data-based modelling structures the data in a specific logical way [6]. Ontology models also contain semantic information to provide a basic meaning of the data and describe their internal relationships [7]. Knowledge graph models provide a framework for data integration, processing, analytics, and sharing as a collection of interlinked descriptions of entities-objects, events, or concepts $[8,9]$.
Figure 1 shows the emerging trend of research papers related to ontologies. As can be seen, the technology appeared around 2002, and the rapidly increasing number of publications in the topic knowledge graphs confirms its success and wide applicability range.

Because of the importance of horizontal and vertical integration in Industry 4.0, ontologies are being used in production systems to share information over an increasingly wide range [1]. Manufacturing companies are faced with many information sharing tasks such as B2M, M2M, and B2B (communication channels between business (B) and/or machine (M) units) $[10,11]$. The information has to be transferred between information systems, optimization methods, or digital twin simulators [12]. Due to the growing demand, the previously proposed developments for ISA-95, ISA-88, AutomationML (Automation Markup Language), and B2MML (Business to Manufacturing Markup Language) industry standards as well as frameworks have intensified [1]; moreover, these are based on knowledge graphs or ontologies.

During the fourth industrial revolution, new methods emerged to deal with this problem, and it can be stated that ontology modelling [13] and knowledge representation are part of the future trends [14], which can be 


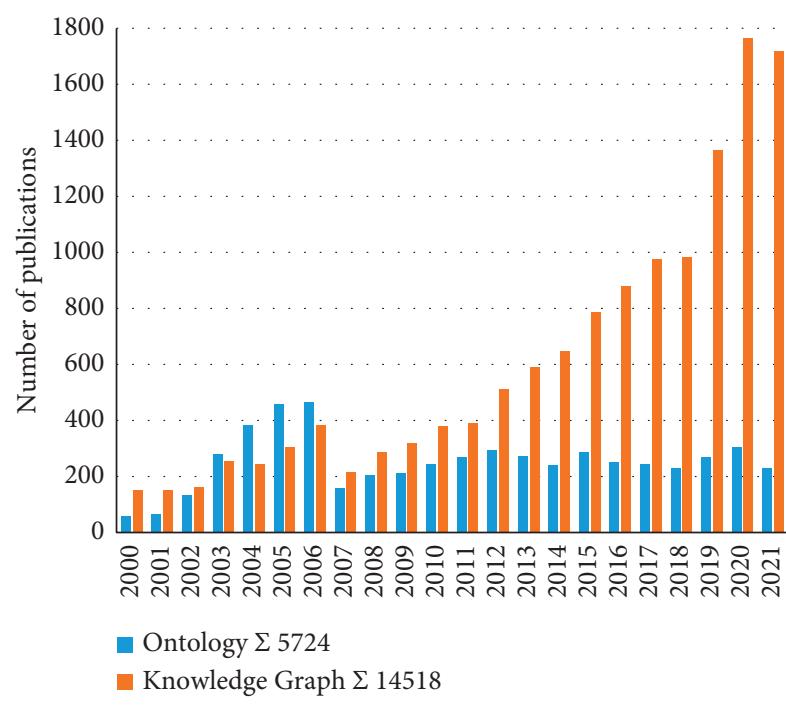

Figure 1: Number of publications since 2000 in the topic of ontology and knowledge graph.

presented using knowledge graphs. A knowledge graph is a programmatic method that subject-matter experts use to model a knowledge domain using data interlinking and machine learning algorithms. Its tasks refer to removing noise, inferring missing information, and determining which facts should be included in a knowledge graph [15]. Also, new collaboration groups are forming as the Industrial Ontology Foundry (IOF) [16, 17] or the European H2020 project OntoCommons: ontology-driven data for industry commons [18] in order to standardize and to support the industry with advanced data interoperability, using reference ontologies. Another problem is the need to improve the efficiency of assembly processes in the manufacturing industry, where computer-aided process planning (CAPP) is gaining importance, which aims to identify appropriate resources while minimizing the total assembly time [19]. This is similar to the emerging importance of resource allocation and process planning in the modern industry [20].

Studies of Gartner Hype Cycle for Emerging Technologies, 2020 [21, 22], predict that ontologies and graphs as technological solutions are going to be available in two to five years. However, these are classified under the trough of disillusionment section, which means these technologies require special precautions to be applied effectively. Ontology modelling can be used for BPR (business process reengineering) or the development of control systems. Furthermore, it is of great importance as a form of system modelling in the concept of Industry 4.0 [23] and digital twin simulations [9]. The importance of this field is also proven by the development of RAMI 4.0 [23] and the other most widely used industrial system models that are characterized such as ADACOR (ADAptive holonic COntrol aRchitecture) for distributed manufacturing systems [24].

The challenges of ontology modelling and analysis of manufacturing processes are summarized as follows: (i) Data processing, structurization, and interoperability

(ii) Standardization in the industry

(iii) Modelling of manufacturing processes

(iv) Horizontal and vertical integration

(v) Share information

(vi) Knowledge representation

(vii) Improve the efficiency of assembly processes

(viii) Process planning

One of the central questions of the digital transformation is how a production system can be utilized to fulfil all the requirements of Industry 4.0 while following state-of-the-art developments. The purpose of our article is to draw attention to each difficulty, and the proposed case study guides as to how these solutions can be effectively applied. We provide an overview of the state of ontologies and standards of manufacturing modelling. We present a well-documented open-source OWL-XML (Web Ontology Language-Extensible Markup Language) based wire harness manufacturing benchmark. The presented case study has already been used to verify production flow analysis [25] and an assembly line balancing problem [26].

This work aims to precisely describe the ontologies of manufacturing processes through a detailed case study and provide guidance on development for researchers and engineers to apply this method and identify further R\&D potentials. Therefore, we present an effective development methodology suitable for developing production-related ontologies and knowledge graphs. As Figure 2 shows, the methodology consists of the following steps:

(I) Data collection:

(i) Find the relevant quantitative and qualitative factors of the manufacturing process, and then appropriately collect the data.

(ii) Perform preprocessing on the raw data and transform the production data into ontologybased datasets.

(II) Ontology modelling:

(i) Establish the basic structural network of the production process and include interactions between groups/classes.

(ii) Determine descriptive and influential factors of the system as cost parameters, requirements, or optimizable elements.

(iii) Develop the desired ontology using appropriate software and connect datasets of production processes with the developed ontology.

(III) Advanced manufacturing analytics:

(i) Analyze the previously defined descriptive and influential factors with data queries and analyses. 


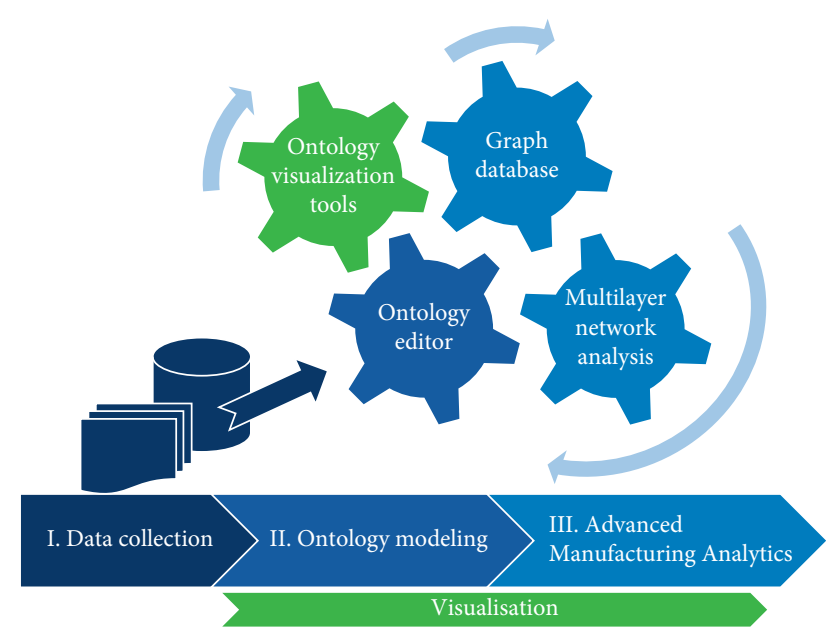

Figure 2: The main steps of the developed methodology.

(ii) Because of the outstanding network analysis and visualization capabilities, it is worth generating labelled multilayer networks from these ontology models and graph databases [25, 27].

Additionally, an essential step of our methodology (also highlighted in Figure 2) is the application of visualization tools that can be beneficial during ontology modelling and advanced manufacturing analytics phases as well. Appropriate visualization of the created ontology with a UML (Unified Modelling Language) or graph diagrams can support the development process or provide additional internal information about the manufacturing procedure. In Section 3, the applied software modules regarding the entire manufacturing-based ontology development methodology are presented.

The novelties of this paper are the following:

(i) In Section 2, the formulation of the main problem is introduced, including the most relevant standardised elements of the manufacturing industry as well as the main features of semantic and ontological modelling technologies. Furthermore, several application examples of semantic and ontology technologies are studied.

(ii) Section 3 introduces an ontology development methodology with a wire harness assembly benchmark.

The applied software solutions and the applicability of a multilayer-network-based analysis approach are demonstrated.

(iii) In Section 4, the results and final contributions are presented in detail.

\section{Elements of the Developed Ontology- Based Framework}

In this section, following the steps of our methodology (see in Figure 2), we present an overview of the relevant standards and modelling methods that are used nowadays in industrial practice such as ISA-95 in Section 2.1; the semantic and syntax elements and the most commonly used ontologies in the manufacturing industry are given in Section 2.2; and finally, ontology-based solutions for production are presented in Section 2.3.

\subsection{ISA-95-Based Standardised Representation of} Manufacturing. The application of a standard can improve the enterprise and manufacturing processes of a company in many ways, such as by reducing costs, enhancing how efficient the flow of information between stakeholders and different enterprise levels or human and physical segments is, and handling data management challenges [28]. Figure 3 summarizes the relevant international standards available in connection with smart manufacturing, which is categorized according to their fields of application. At the intersection of Business and Supply Chain Logistics and Manufacturing Operations Management (Figure 3), the ANSI/ISA-95 (IEC 62264 from the International Electrotechnical Commission) standard is found, which uses a five-level hierarchical control model to represent the Business Logistics, Manufacturing Operations Management, Production Control, and Production Process functions [29, 30]. It is a widely used international standard produced by the International Society of Automation for developing an automated interface between enterprise and control systems [1]. ISA-95 can be potentially related to the creation of a manufacturing process ontology or knowledge graph, such as

(i) Product/process/model hierarchy

(ii) Product capability model

(iii) Role-based equipment hierarchy

Different model parts from IEC 62264 are linked together logically to define the hierarchy of submodels as shown in Figure 4. The production information defines what was made and used in the process, that is, which elements correspond to information during production scheduling that listed what was to be made and used. The production scheduling elements correspond to the product definition that shows what is specified to make a product. The process segment descriptions are defined by the product definition elements that prove what can be done with the production resources according to the information available. Process Specification and Production Capability prove the main information about the resources [31].

Figure 5 shows a UML (Unified Modelling Language) [32] diagram describing the Production Capability model regarding IEC 62264, and the information from subclasses represents the capability and capability property characteristics of Personnel, Equipment, and Materials. There are several new methods/frameworks for the standardization and modelling of modern production automation such as IICF (Industrial IoT Connectivity Framework), IIRA (Industrial Internet Reference Architecture) [33], NIST (National Institute of Standards and Technology) [34], and RAMI [35]. The basic principle of these methods is similar to the ISA-95 standard; it organizes the production/ 


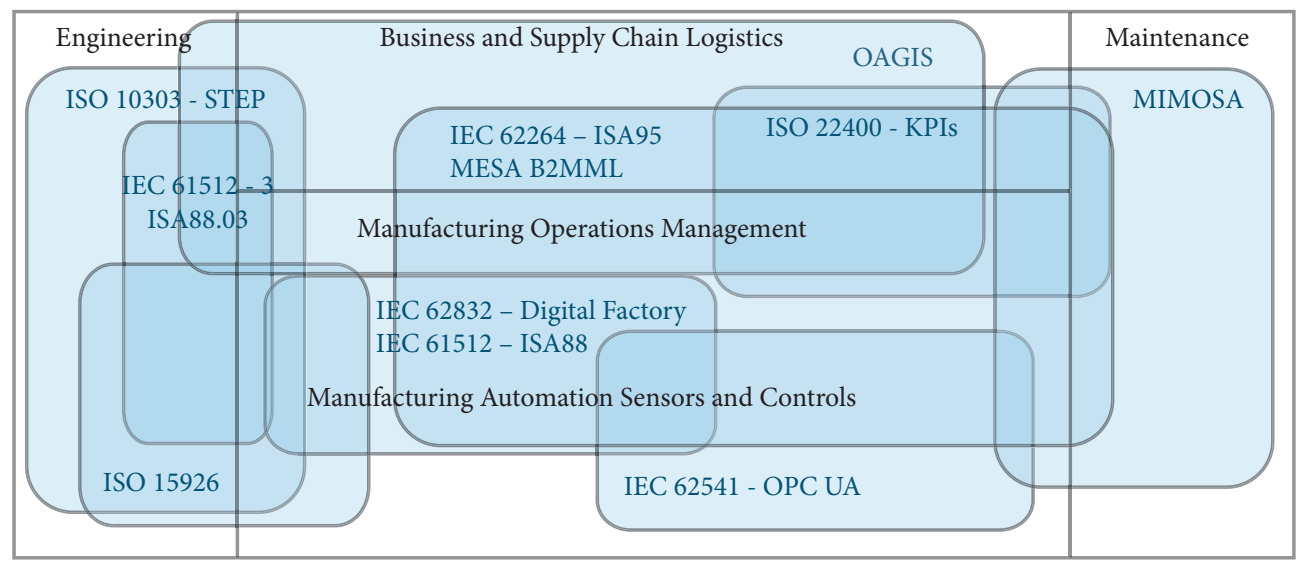

FIGURE 3: A summary of international standards for smart manufacturing [28].

manufacturing processes into a unified hierarchy, allowing for the appropriate communication of information.

Integrating the industrial aspects with linked data and semantic technologies leads us to a knowledge graph solution. We will continue with semantic modelling in the following section.

\subsection{Semantic Modelling, Ontologies, and Description Methods} of Manufacturing Systems. In this section, our goal is to provide a simple overview of describing the previously mentioned production standards using the most critical elements of semantics and syntax. This section provides the necessary theoretical background to understand the specific semantic models used in ontologies and description methods of a manufacturing system. Furthermore, we summarize the most relevant ontological methods amongst others to describe production systems within the scope of Industry 4.0. The RAMI and the AutomationML framework are discussed more widely as the literature review shows that leading state-of-the-art research is related to RAMI.

The Semantic Web Stack (see Figure 6) illustrates the hierarchy of (web) languages, where each layer uses the capabilities of the layers below it. It represents how technologies (which are standardised for the Semantic Web) are organized to make the Semantic Web possible [36]. A realistic architecture for the Semantic Web must be based on multiple independent but interoperable stacks of languages $[37,38]$. Ontology and knowledge graph development require these synergistic syntaxes in the same way.

The main principles are the RDF (Resource Description Framework) [39] and OWL (Web Ontology Language) [40]. The RDFS (RDF Schema) provides interoperability between applications that exchange machine-understandable information on the web. It has a wide range of applications, for example, resource discovery to provide better search engine capabilities or to describe the content and content relationships available on a particular website. OWL can develop domain-specific schemas and ontologies (so-called metamodels) and represent the meaning of terms in vocabularies and the relationships between such terms.
RDF triples can be utilised to extend a graph between unique data instances, collect general data, and express semantics, attributes, and hierarchies [41]. Another principle uses URI (Uniform Resource Identifier) to link data by creating triple sentences with subjects, predicates, and objects [42]. Figure 7 shows a visualised example. The operator plays the role of the subject, which has a data property link (about) to additional data concerning the operator data, skill, or ID. Furthermore, information about the subject is provided by a predicate that is linked to an object using an object property, so the RDF operator (subject) assigns an operator (object property) to the activity (object).

Nowadays, the most utilised ontology language for Semantic Web applications is OWL 2 [43], the structure of which is illustrated in Figure 8. The main building blocks of OWL 2 are various concrete syntaxes that can be used to serialise and exchange ontologies. Each part of the Semantic Web Stack (Figure 6) can be accessed with OWL 2. Therefore, we regard the application of this language as a highly versatile and well-applicable development method for ontologies. An optional connection is highlighted by the Manchester syntax in Figure 7, which stands for the capability of OWL 2 to write database queries using SPARQL (Structured Protocol and RDF Query Language) to manage knowledge explorations in ontologies [44].

Standards have significant importance for realizing the Industry 4.0 vision and industrial digital chain monitoring to reduce costs. There are several studies $[45,46]$ concerning the management of Industry 4.0-related standards and terminologies as well as the creation of knowledge-based frameworks. A good overview is provided by an ontology called Industry 4.0 Knowledge Graph [47], which has been developed in order to represent and categorize standards, standardization organizations, and standardization frameworks involved in the domain of Industry 4.0 [46]. In Figure 9, the complexity of this field is highlighted. Different domains are connected to the RAMI 4.0 and Asset Administration Shell [48] such as Hierarchy Levels, Communication Layers, Engineering, and Semantics.

The Asset Administration Shell has also been established to provide a digital representation of all related information and services involved in manufacturing components $[35,49]$. These 


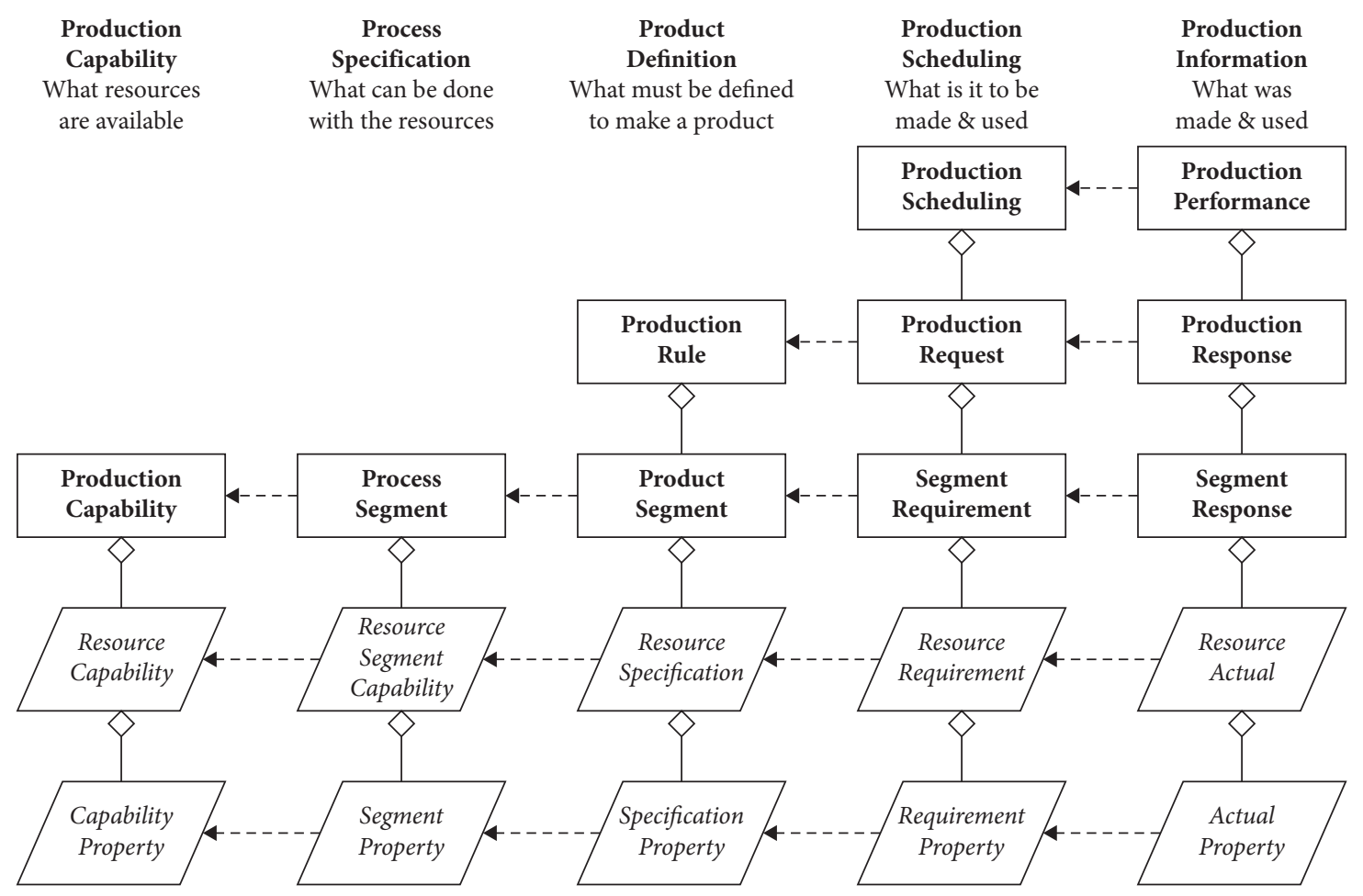

FIgURE 4: The IEC 62264 hierarchy model (IEC 62264) [31].

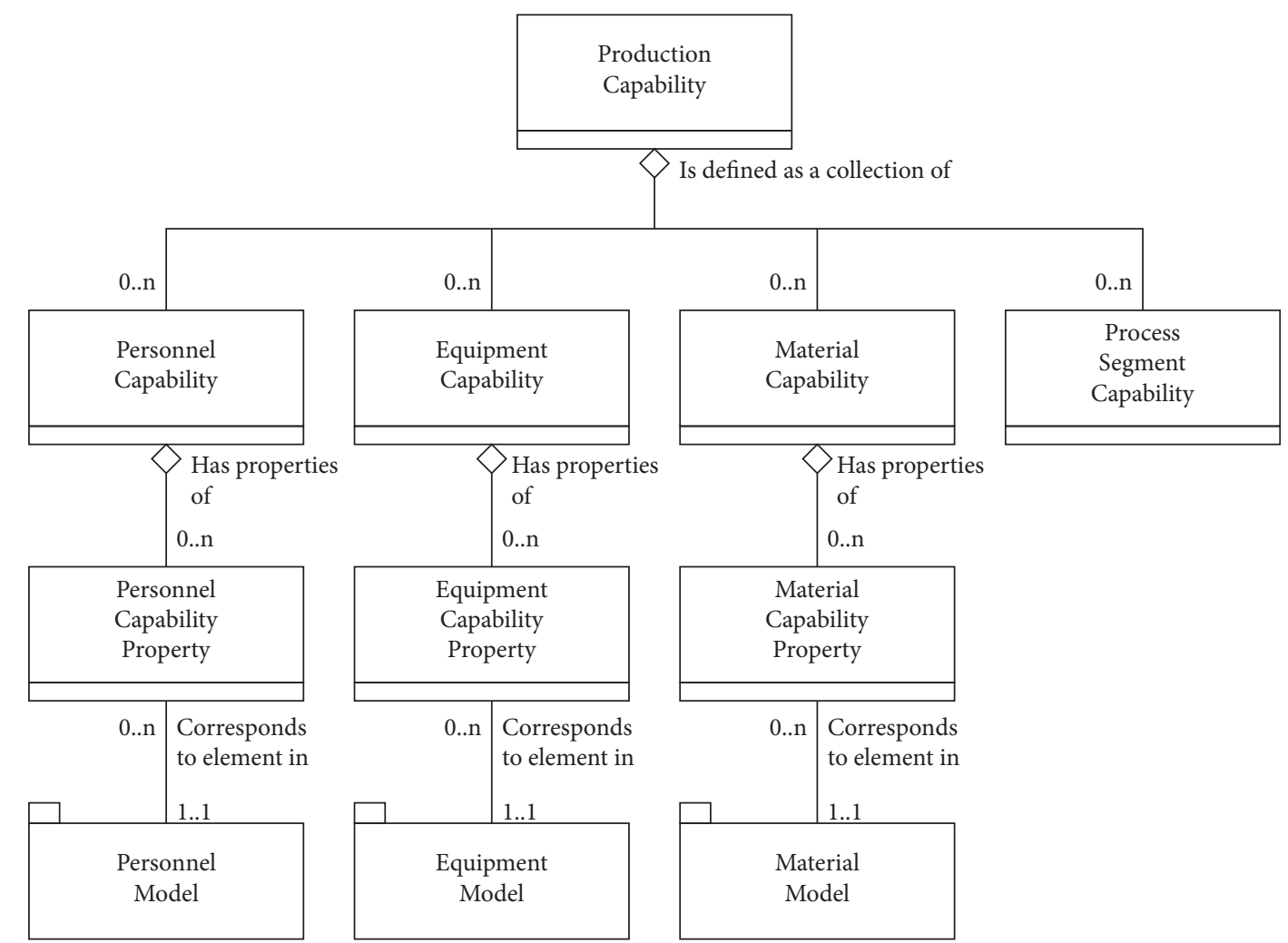

Figure 5: The Production Capability model (IEC 62264) [31].

layers are listed and categorized in Figure 9. In this study, we focus on Semantics and Hierarchy as building blocks of RAMI 4.0 and the Asset Administration Shell, as is highlighted in red in the figure. The Semantic Web Stack of the W3C (World Wide Web Consortium) is a significant building block of the RAMI 4.0 system (see Figure 9). 


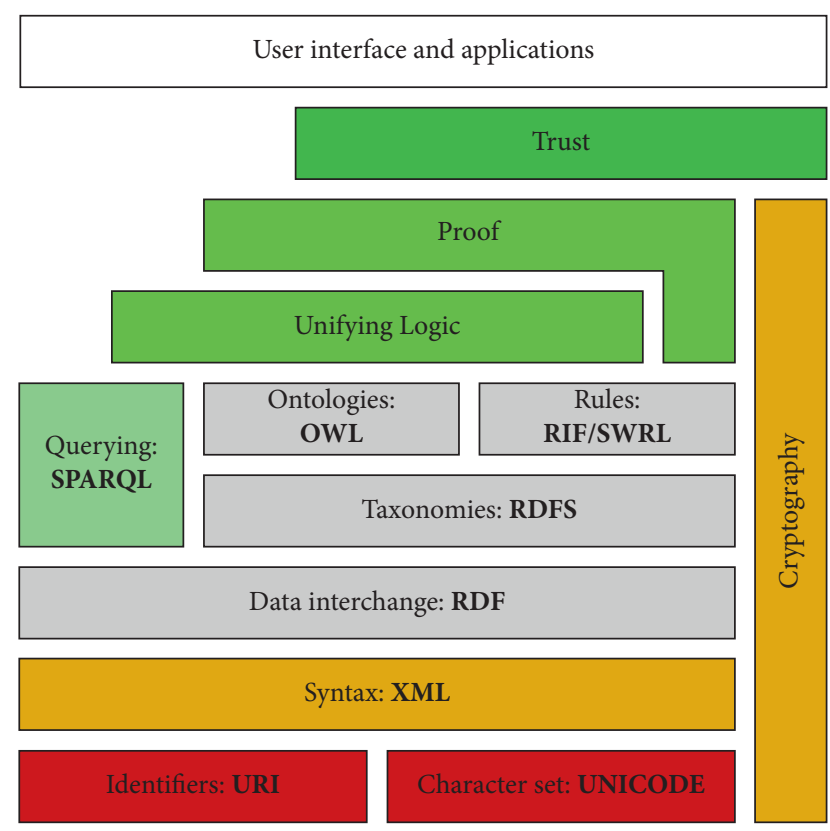

FIgure 6: The Semantic Web Stack [36].

Furthermore, we would like to show some examples to prove the importance of the semantic representation of industrial standards and processes. Therefore, we have summarized the studied semantic and descriptive methods in Table 1.

In the modern industry, one of the most widely used frameworks to describe components of Cyber-Physical Systems (CPSs) from various perspectives is the AutomationML standard [50]. An open, XML-based data exchange format aims to ensure consistent and lossless data exchange in the design of manufacturing systems. It enables systems to be modelled from single automation components to entire large and complex production models. It supports the representation of various aspects of the system, namely, topology, geometry, kinematics, and control behaviour [50]. In addition, AutomationML methods are capable of modelling IEC 62264-2-compliant information also [60]. Furthermore, a specific ontology has also been developed, namely, the AutomationML Ontology (AMLO), to provide a semantic tool for the improvement of engineering processes in the design of CPSs [51].

The Sensor, Observation, Sample, and Actuator (SOSA) ontology provides a core for the Semantic Sensor Network ontology (SSN) as well as extends the target audience and application areas, making use of Semantic Web ontologies. It has been used as part of an architecture for the Web of Things, sensing in manufacturing, and representing humans and personal devices as sensors, as well as part of a linked data infrastructure for SWE (Sensor Web Enablement) [52].

The Manufacturing Resource Capability Ontology (MaRCO) supports the rapid semiautomatic system design, reconfiguration, and autoconfiguration of production systems. MaRCO has been developed for the quick identification of candidate resources and resource combinations for a specific production need [53]. IoT-O is a core-domain

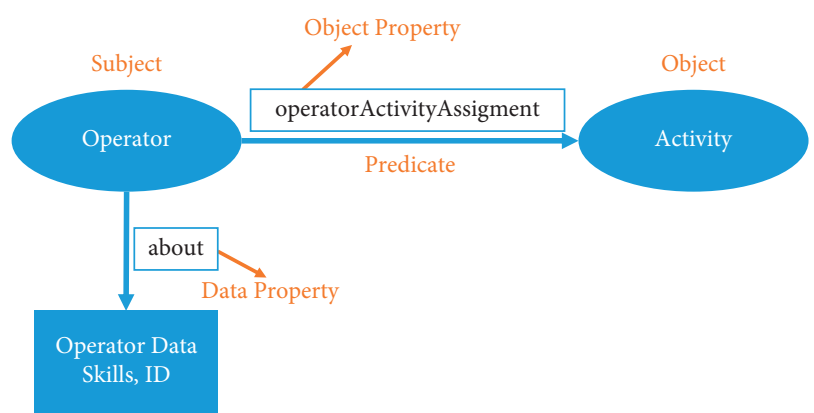

Figure 7: Theoretical example of an RDF triple in an RDF model.

modular IoT (Internet of Things) ontology that proposes a vocabulary to describe connected devices and their relationship with their environment. It describes concepts such as electronic device, smart network, smart entity, physical entity, and control entity [54, 61].

Upper ontologies can define top-level concepts such as activities, physical objects, or topological relations from which more specific classes and relations can be defined. Upper ontologies are used to develop a more specific domain ontology by starting with the identification of crucial concepts utilising activity modelling, use cases, and competency questions [62]. In the following, we look through the more relevant upper ontologies concerning manufacturing systems.

The Basic Formal Ontology (BFO) serves as an upperlevel framework, which has been developed to assist the organization and the integration of data obtained through scientific research $[55,63]$. Furthermore, BFO is currently undergoing a certification process with the International Organization for Standardization (ISO) as a top-level ontology for information technology [64].

The Assembly Sequence Planning (ASP) ontology formally defines the assembly knowledge, where all the assembly knowledge in the sequence generation approach is expressed and stored. ASP determines the sequences and paths of parts to assemble a product with minimum costs and over the shortest period of time $[56,65]$.

Manufacturing Service Description Language (MSDL) provides the simple building blocks required to describe a broad spectrum of manufacturing services. MSDL is a description of the manufacturing capabilities of manufacturing resources at different abstraction levels, namely, machine, workstation, cell, shop, and factory [57].

A widely used ontology with many extensions is the Descriptive Ontology for Linguistic and Cognitive Engineering (DOLCE). The DOLCE upper ontology aims to capture the ontological categories underlying natural language and human common sense, which is of great importance in terms of the Semantic Web [58]. DOLCE + DnS Ultralite (DUL) is the OWL version of DOLCE, extended to cover the Descriptions and Situations (DnS) framework, and is a widely adopted ontology in projects worldwide. The foundational concepts of DOLCE can be utilised for aligning domain ontologies, e.g., for Semantic Sensor Networks [66]. DUL is also used for creating a formal model of events to 


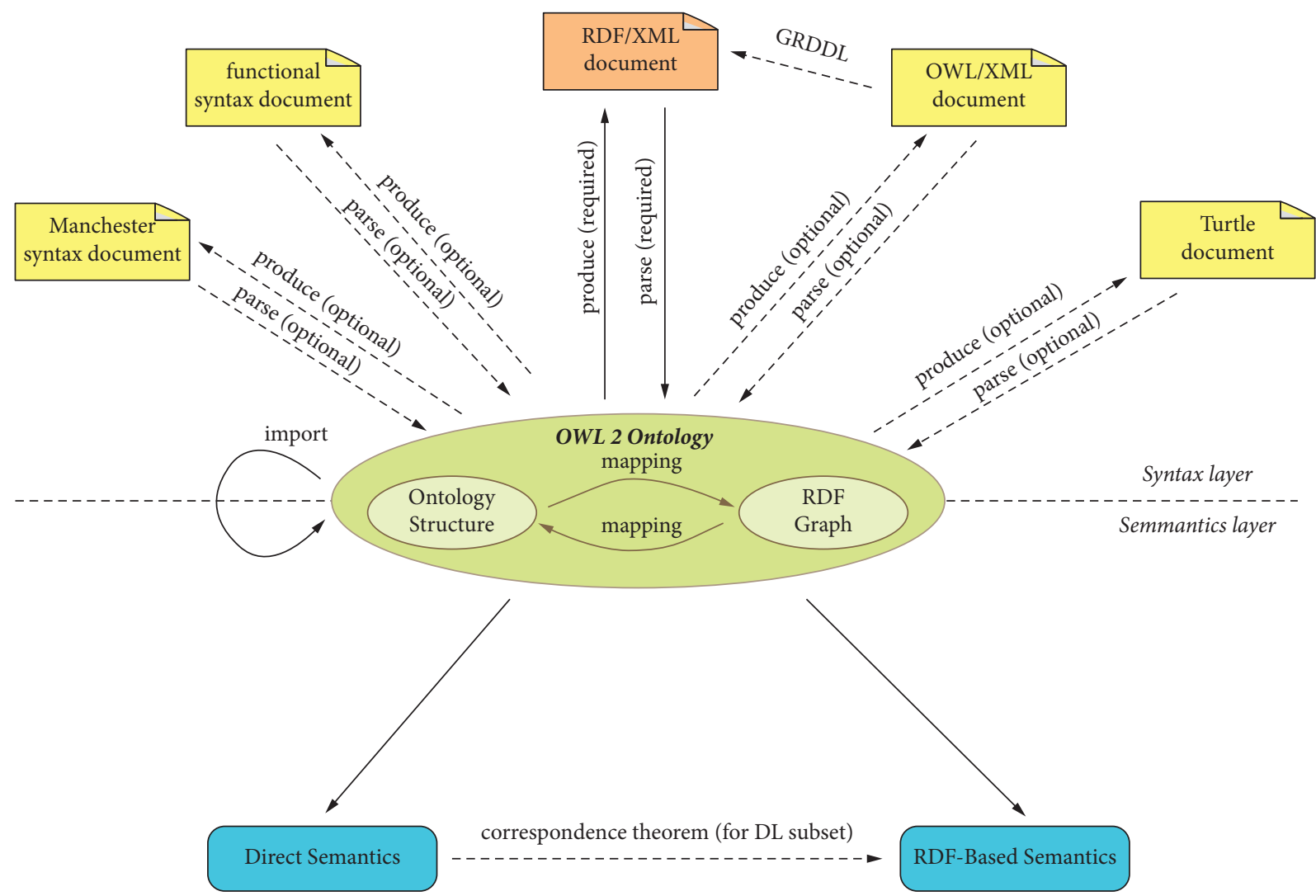

FIgURE 8: The structure of OWL 2 (Web Ontology Language) [43].

provide comprehensive support to represent time and space, objects, and people, as well as causal or correlational relationships between events [59]. With another DUL application, the extraction and description of emerging content ontology design patterns are achieved [67].

In summary, ontologies play a vital role in developing smart factory concepts [68], and the application of their elements as namespaces or vocabularies in specific manufacturing-related ontologies is justifiable.

\subsection{Ontology-Based Analysis and Solutions in Manufacturing} Systems. In this section, we show, through a couple of applications, that ontology- and knowledge graph-based solutions for production are no longer just concepts but methods that have already been implemented in the industry.

A research group of ABB Company proposed a study about a scheduling solution connected to a production environment aligned with the ISA-95 standard and using B2MML to share information. The methodology of the ontology data-supported workflow is shown in Figure 10 [69]. The development of the Enterprise Control Ontology (ECO) [70] also exemplifies well that semantic models provide various solutions to address operational issues in production. In ECO, more subontologies have been combined to create a manufacturing system model, which provides domain information about entities and enables the reconfiguration of manufacturing systems.
Another application example is the SemCPS framework (Semantically Described Cyber-Physical Systems) for enabling the integration of CPS descriptions in knowledge graphs. The approach can effectively integrate CPS perspectives using Uncertain Knowledge Graphs of smart manufacturing-related standards such as AutomationML [71]. Numerous application and research examples can be found for AutomationML-related developments, such as data modelling and Digital Twin Exchange $[9,72]$, or IEC 62264 standard-based AutomationML models can be created [73]. Another important aspect is to implement the Bill of Process (BOP) and Bill of Materials (BOM) in data models to map resources or abilities in order to perform a task based on physical skills [74]. The integration of BOP and BOM in resource description models can support assembly planning engineers and provide a framework for clustering production information [75].

The Uniform Project Ontology utilizes linked data and the Semantic Web in order to represent knowledge about megaprojects to facilitate data processing and utilisation through their entire lifecycle [42]. The purpose of a recently published modular domain ontology is to describe the cyber and physical aspects of automation systems that support simultaneous engineering [12]. The effectiveness of the knowledge-based approach to designing assemblies in agile manufacturing to integrate a linked product and process data has been studied and proven [76].

A study by Siemens has proven that semantic technologies can improve the feature selection method for machine 


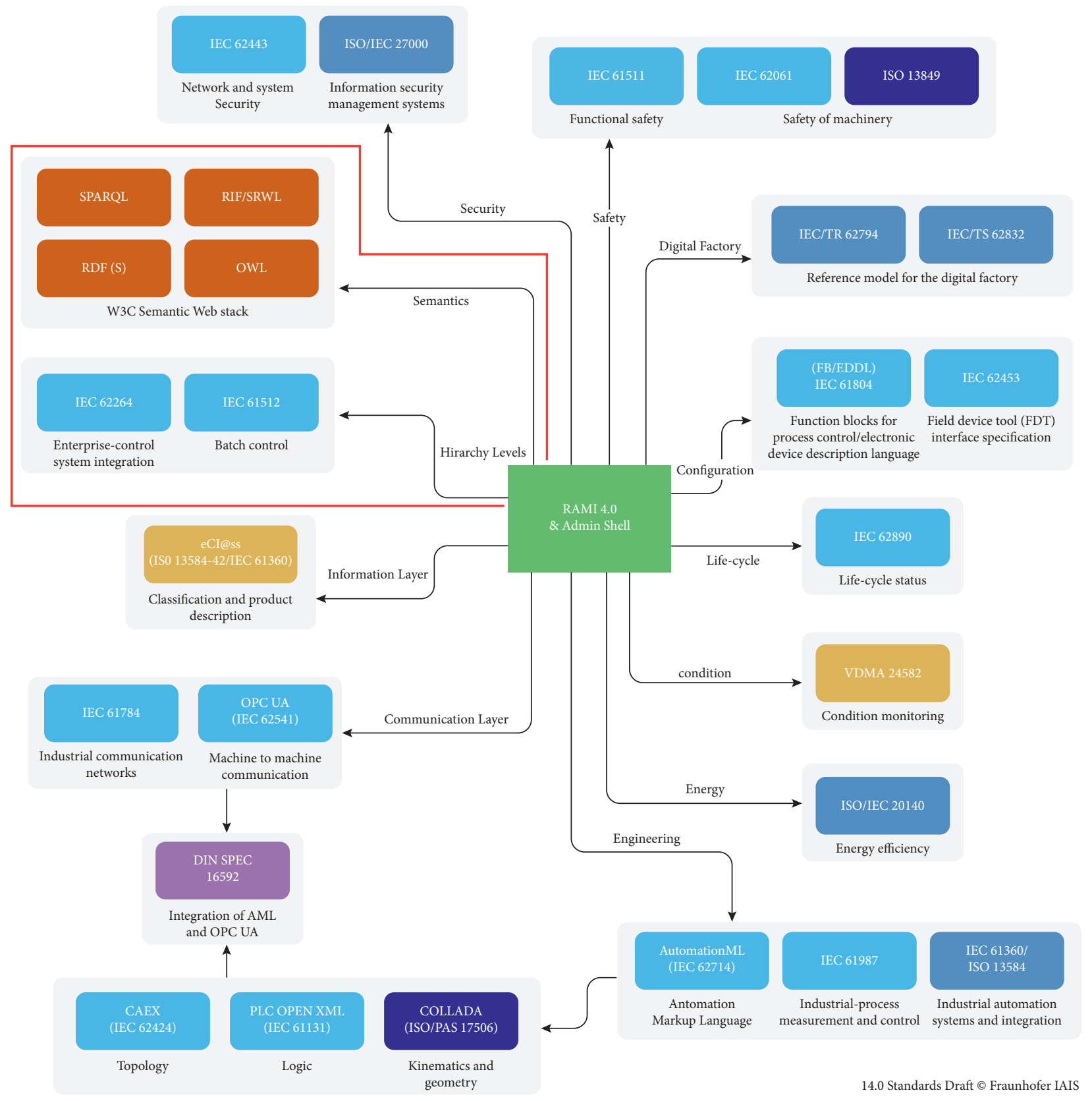

FIgURE 9: Industry 4.0 standards by means of semantic technologies [46].

learning models in industrial automation systems in order to reduce the size of feature spaces for exemplary learning problems using only a small amount of semantic relations [77]. During the Optique program [78], the goal was to develop an Ontology-Based Data Access (OBDA) system and provide access to Industrial Big Data stores using Semantic Web technologies. An impressive demonstration of using Optique's OBDA system customized for the user is Siemens Energy's data access challenge. The semantic technology was used to answer questions concerning data queries as follows: "return the top 10 errors and warnings for turbines of product family X" or "which events frequently occur before a specific point in time?" [79]. A further project in collaboration with Siemens studied the application of ontologies to create industrial information models in manufacturing and energy production. It led to the development of the Siemens-Oxford Model Manager (SOMM) tool to support engineers in creating ontology-based models [80].

The applicability of a graph-based framework for Advanced Manufacturing Analytics has also been demonstrated by representing manufacturing data as a multigraph using a semantic abstraction layer to integrate flexible data. Herewith, a tool is provided for predictive and prescriptive decision-making by detecting fault patterns [81].

In a previous study of the authors, the assembly line balancing problem was investigated using a multilayer network modelling method, and the interacting elements of the 
TABLE 1: List of the most relevant ontologies and description methods in manufacturing.

\begin{tabular}{lc}
\hline Name & Short description \\
\hline I40KG [47] & Industry 4.0 Knowledge Graph \\
$\begin{array}{l}\text { AutomationML } \\
\text { [50] }\end{array}$ & A standardised XML-based Automation Markup Language, which aims to store and exchange the information of \\
plant engineering & Plo [51] \\
SOSA and SSN [52] & AutomationML Ontology, which covers the Computer-Aided Engineering Exchange (CAEX) section of the standard \\
SWE [52] & Sensor, Observation, Sample, and Actuator and Semantic Sensor Network ontologies \\
MaRCO [53] & Sensor Web Enablement, which is a suite of standards that has been developed \\
IoT-O [54] & Manufacturing Resource Capability Ontology \\
BFO [55] & Internet of Things Ontology \\
ASP [56] & Basic Formal Ontology \\
MSDL [57] & Assembly Sequence Planning Ontology \\
DOLCE [58] & Manufacturing Service Description Language \\
DUL [59] & Descriptive Ontology for Linguistic and Cognitive Engineering \\
\hline
\end{tabular}

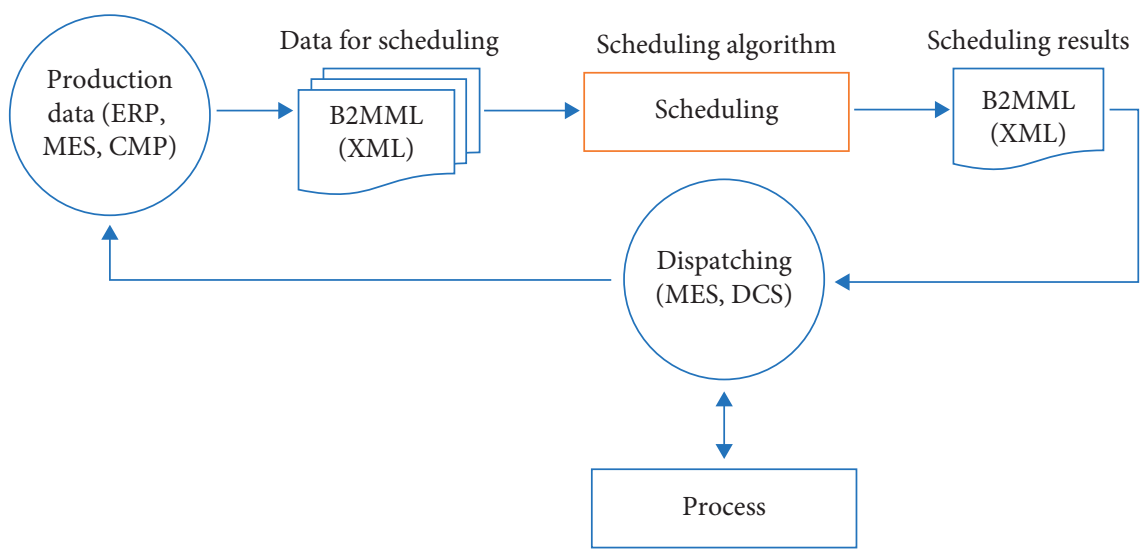

FIGURE 10: Flexible scheduling supported by production ontology data [69].

assembly line were mapped as bipartite graphs [26]. The methodology of how links data with RDF triples (discussed in Section 2.2) is the same as in the case of bipartite graphs. Studies are published to formalise the bipartite graphs as an intermediate model for RDF with a goal of graph-based notions in querying and storage [82]. Furthermore, an RDF database can be simultaneously analyzed as layers of a multilayer network [83], providing a solution for production flow analysis.

Based on the above-listed application examples, the benefits of the ontology-based features are the following:

(i) Support flexible scheduling and solve operational issues in manufacturing, such as resource allocation $[69,70]$

(ii) Integrate CPS and describe cyber and physical parts of automation systems [71]

(iii) Model digital twins and provide access to Industrial Big Data stores $[9,72]$

(iv) Conceptual design of a data model and warehouse [73-75]

(v) Facilitate project lifecycle analytics of megaprojects [42]

(vi) Effectively support assembly design and planning processes to evaluate risks and costs before the realization of the production processes $[12,76]$ (vii) Improve the efficiency of machine learning models [77]

(viii) Data mining and root cause analysis $[78,79]$

(ix) Support predictive and prescriptive decisionmaking $[80,81]$

(x) Supplementary technique to solve the assembly line balancing problem [26]

In the previous sections, we proposed and summarized the results of research on the ontology-based modelling of production systems and collected the most relevant application cases. In the following section, we will present the applicability of our methodology in a wire harness assembly case study.

\section{Ontology-Based Wire Harness Assembly Benchmark}

This section describes our developed methodology (which has been briefly presented at the end of Section 1 and in Figure 2), more detailed with the applied software elements in Section 3.1. After that, based on the steps of our methodology (see in Figure 2), we present a detailed industrial benchmark, starting with a use case from the wire harness manufacturing industry in Section 3.2. The ontology-based 
modelling and the development of a knowledge graph are described in Section 3.3. Finally, the data queries with SPARQL and the multilayer visualization with data analyses are presented in Section 3.4.

3.1. Applied Software Tools for the Developed Methodology. Firstly, we are detailing different software features utilised in our method, represented in Figure 2. The main theoretical steps of our development methodology have been described already in Section 1. The applied software packages involved in our work are as follows:

(i) Protégé for ontology development and to create the OWL/RDF format [84]

(ii) GraphDB to manage SPARQL queries [85]

(iii) MuxViz to visualize the internal data and manage advance manufacturing analytics [86]

(iv) OntoGraph and VOWL plugins of the Protégé environment to visualize the ontology structure

Figure 11 represents the three stages of the data processing in our methodology. The first step is to transform the collected data from the production system into ontologybased datasets (linked data as RDF). Then, after the ontology skeleton is final (also referred to as system modelling), the production datasets are connected with the ontology. During the third phase, the created RDF-based semantic network is turned into a multilayer network. To perform these tasks, we utilised methods based on vector- and matrix-sorted data aggregation.

In the case of data collection of a wire harness manufacturing, we studied a modular assembly system. The relevant data are the physical parts of the final product, stored in the BOM, the operators at the assembly line and their skill, and the necessary equipment. Quantitative factors are the activity times of a particular step of the wire harness assembly and the costs of using different skills or resourcerelated tools.

For the development of the ontology, we used Protégé, which is an open-source tool developed by Stanford University to create and edit any ontology [87]. This platform supports all kinds of semantics and data standards such as the XML, RDF, or OWL types of ontology datasets [88]. Indepth knowledge of the behaviour of the structure of the manufacturing system is required to assign which factors or identities will be a class, object property, or data property in the formed ontology. For this reason, the hierarchy of manufacturing processes must be adequately reflected using the tools provided by the ISA-95 standard or AutomationML framework (as presented in Section 2.2). Another critical part of ontology engineering is to find the reusable Ontology and Vocabulary elements, which can be applied in the current ontology. To accomplish this, firstly, industryspecific research papers and semantic solutions (studied in Section 2.2) can provide a guideline, but also the use of the tool Linked Open Vocabularies (LOV) is recommended [89] as it provides an effective search engine to find adaptable namespaces or vocabulary elements.

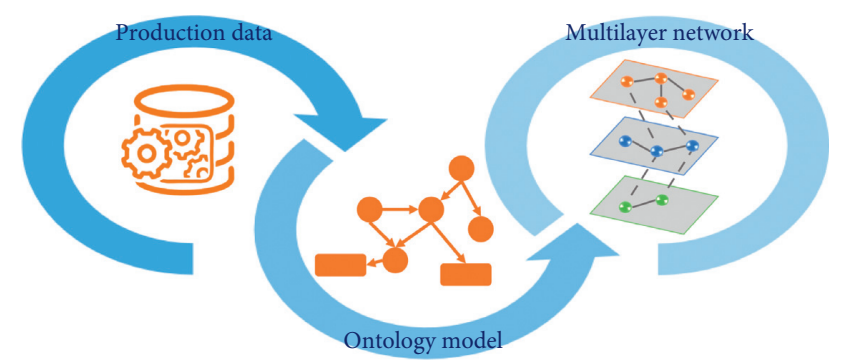

FIgURE 11: The steps of data transformation toward creating a process-specific ontology and multilayer network.

For the advanced manufacturing analytics part of the methodology, we utilised several solutions. For data analysis and SPARQL queries, we used GraphDB software, an RDFcapable database tool, especially for knowledge graphs. The most significant benefit of SPARQL queries is creating a structured version from the data stored in the ontology or extracting data from RDF, which is an excellent source to manage basic production analysis. Furthermore, the MuxViz software application $[86,90]$ has been used to create multilayer graph representations to explore further analytical possibilities. However, if a more in-depth investigation is needed or the production ontology is of high complexity, the tools of Data Science can provide more accurate solutions.

Additionally, visualisation tools play an essential part in our methodology in several phases of the process. We utilised OWL visualisation tools, which are part of Protégé [91] as the VOWL (Visual Notation for OWL Ontologies) [92]. Furthermore, we utilised MuxViz as well to create multilayer graph representations and to networks analyse. By graphically examining process networks, we can identify and analyze different inhomogeneities. Identifying the core nodes and investigating the critical edges or node-degree distribution within the network structure can provide internal information about the production process. The multilayer-based approach of manufacturing analytics and the case study, which has been presented, have been used in other papers written by the authors to verify production flow analysis [25] and solve assembly line balancing problems [26]. In the following, we detailed the wire harness manufacturing use case for ontology representation.

In the following sections, we prove the proposed methodology via a manufacturing-specific benchmark.

3.2. Data Collection: A Case Study of Wire Harness Manufacturing. An open-source benchmarking problem of a modular wire harness production system has been developed to support reproducible development. Wire harnesses are produced by a typical complex modular production system [93] . The challenge is that numerous activities and the highly manual assembly necessarily require optimum assembly line balancing [26]. The complexity of wire harness manufacturing is represented by a multilayer network [25] where the basic activities are connected to each layer. The information that needs to be acquired is a precise prediction of the duration of these activities [94], which can be measured by a fixture sensor. 
The following use case definition depends on former works of the coauthors [94]. The manufacturing is modular, which means the products $p_{1}, \ldots, p_{N}$ are built from a set of modules $m_{0}, \ldots, m_{N_{m}}$. The number of types of products $N_{p}$ was 64, and $N_{m}$ was defined as a combination of 7 modules: $m_{0}$ base module, $m_{1}$ as left- or right-hand drive, $m_{2}$ normal/ hybrid, $m_{3}$ halogen/LED lights, $m_{4}$ petrol/diesel engine, $m_{5} 4$ doors $/ 5$ doors, and $m_{6}$ manual or automatic gearbox. $N_{a}$ was defined as 654 activities/tasks categorized into $N_{t}$ which consisted of 16 activity types with well-modeled activity times. These times are based on benchmarks from the literature [95]. Table 5 shows the duration of the main activity types and defines which activity time depends on the number of wires. In these activities, $N_{c}$ was equal to 653 different built-in parts (among these, $C_{r}=299$ terminals, $C_{b}=113$ bandages, $C_{c}=38$ connectors, $C_{d}=155$ wires, and $C_{l}=48$ clips). $N_{z}$ was also defined as 6 zones for the workstations (see Figure 12) to determine where the components are placed on the assembly table.

The assembly line $N_{w}$ consisted of 10 workstations (assembly tables). For every assembly table, one operator is assigned; therefore, $N_{o}=10$. Required $N_{s}$ was also defined as 5 skills of the operators, namely, $s_{1}$-laying cable, $s_{2}$-bandaging, $s_{3}$-attaching the terminal, $s_{4}$-installing the connector, and $s_{5}$-inserting the clip. A piece of equipment is required for every activity; therefore, $N_{e}=5: e_{1}$-cabling tool, $e_{2}$-bandaging tool, $e_{3}$-terminal handler, $e_{4}$-connector handler, and $e_{5}$-clipping tool. Some tools require a resource $\left(N_{r}=2\right): r_{1}$-compressed air and $r_{2}$-electricity.

\subsection{Ontology Modelling: Creation of the Manufacturing-Based} Knowledge Graph. This section presents the modelling part of the ontology development based on the theoretical background presented in Section 2.2. The classes and their interactions are determined regarding the use case of wire harness manufacturing, which is detailed in Section 3.2.

As the first phase of the ontology development, the basic structure has to be established. Therefore, different classes are defined as the elements of this specific production process, as shown in Figure 13. To characterize the relationships between these classes, we distinguish different types of connections as cost, optimizable, or technical parameters. These properties related to interconnections, socalled object properties, significantly determine the characteristics of the production process.

The presented wire harness manufacturing ontology consists of 9 classes: product, module, component, activity, skill, operator, workstation, equipment, and resource (namely, electricity for some tools). Furthermore, interactions between these classes are denoted by arrows in Figure 13, pointing to the domain class of the object property.

Once the theoretical structure of the ontology is available, the following part is the creation of a manufacturing-based knowledge graph, where first, we implement the relevant vocabulary and namespace elements. Table 2 summarizes the namespaces used for the

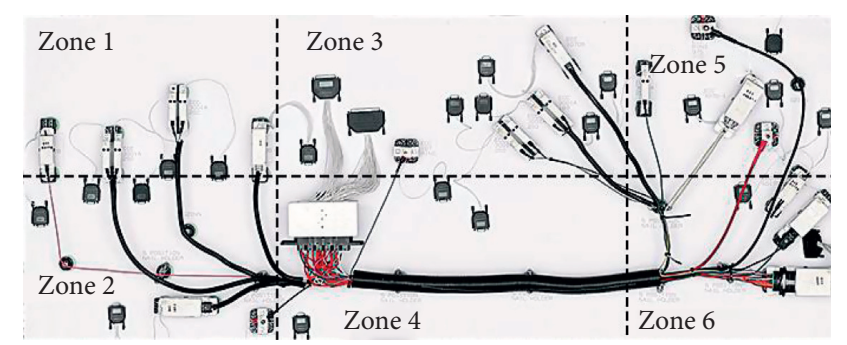

FIGURE 12: Illustration of the distribution of the fixtures on an assembly table and the definitions of the zones. As the fixtures move according to the assembly tables of the conveyor system, the fixtures are identically placed at every workstation.

wire harness manufacturing ontology. The sources of the vocabularies are cited next to their prefixes in the table. Figure 14 represents the structure of the wire harness manufacturing ontology after the integration of vocabulary elements. As listed in the legend, different classes and object properties are denoted by different colours. Furthermore, industry-specific data (described in Section 3.2) have been implemented in the ontology as data properties, which are listed in Table 3 together with the final applied object properties.

The final Protégé implementation of the wire harness manufacturing ontology shown in Figure 15 provides a structural overview of the model in the OWL format. The dark-blue-coloured classes and object properties come from prefixes/namespaces, and the data properties denoted in green are visualised together with their data types. This brings us to the end of the ontology modelling, which can be exported in any $\mathrm{RDF}$ format and proceed with data analysis and queries.

3.4. Advanced Manufacturing Analytics. In modular manufacturing, line balancing is a complex task, especially in manual production processes. The wire harness production is highly required workers for the assembly processes. Usually, the production line contains a special conveyor, namely, rotary lines, where the assembly tables are on the conveyor with several assembly fixtures (see Figure 12). In this section, we created SPARQL queries on the ontology data to analyze the current situation, where the collected manufacturing data are considered (Section 3.2). After that, the RDF-based multilayer network is created to visualize and analyze the connectivity between the individuals. The two methods are evaluated together to discover the potential of the line balancing improvements.

The results of SPARQL queries for production data analysis are shown in Figures 16 and 17. The entire code of SPARQL queries can be found in Appendix A. In the first case (Figure 16), we investigated how much unique component is required for seven different modules from the five distinct types of components as a wire or terminal. It can be stated that the most complex wire harness module is $m_{0}$, which is the base module, with more than 350 components, while $m_{6}$ has the fewest. We can see that 


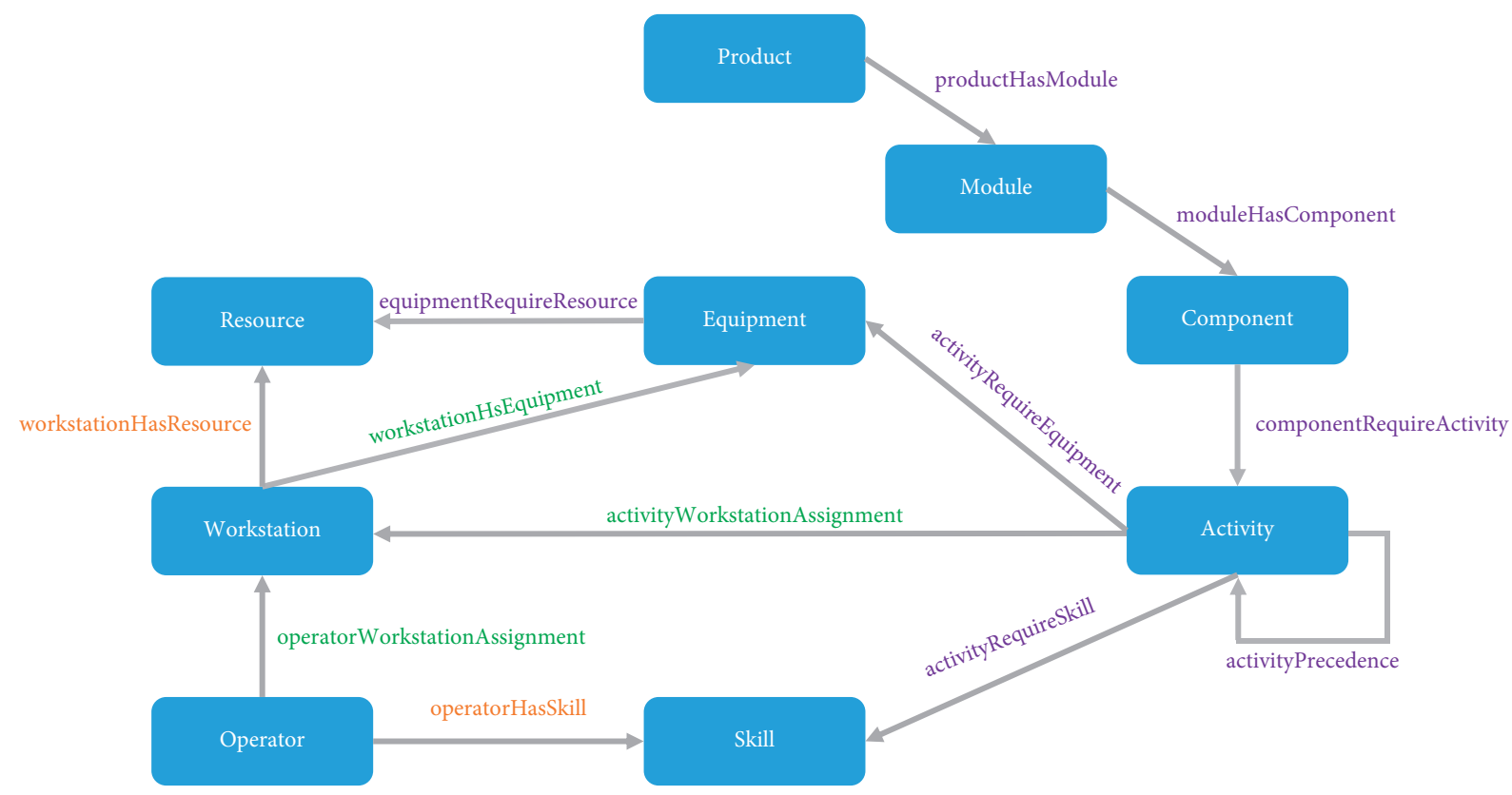

Legend

Cost parameter

Technology requirement

Optimizable parameter

FIGURE 13: Technology and cost-related factors of a wire harness manufacturing visualised on the theoretical ontology.

TABLE 2: List of the integrated ontology namespaces.

\begin{tabular}{l}
$\begin{array}{l}\text { Prefix } \\
\text { RAMI [96] }\end{array}$ \\
$\begin{array}{l}\text { SMO [97] } \\
\text { PROV }\end{array}$ \\
$\begin{array}{r}\text { The PROV Ontology (PROV-O) provides a set of classes, properties, and restrictions that can be used to represent and } \\
\text { interchange provenance information or data coming from different systems and in different contexts }\end{array}$ \\
$\begin{array}{r}\text { SCOR [99] } \\
\text { The vocabulary SCORVoc formalizes the latest SCOR (Supply-Chain Operations Reference) standards while overcoming the } \\
\text { identified limitations of existing formalizations }\end{array}$ \\
$\begin{array}{r}\text { DUL [100] } \\
\text { DOLCE + DnS Ultralite ontology aims to provide a set of upper-level concepts that can form the basis for easier } \\
\text { interoperability among middle- and lower-level ontologies }\end{array}$ \\
\hline
\end{tabular}

the number of different components is evenly distributed in each module, so terminals are the most, and connectors are the fewest in every module. As mentioned in Section 2.3, the implementation of the Bill of Materials in the ontology (or data model) may yield valuable information, which can support the work of process engineers or designers. The analysis of the assembled components per module is critical toward discovering the relevance of the module to get more precise production scheduling.

In the second graph (Figure 17), the most complex product $\left(p_{64}\right)$ is investigated. All seven different modules are involved in this wire harness product, and the entire assembly process is distributed over ten workstations. How much built-in component-related activity is assigned to each workstation to assemble this product is highlighted. The figure also summarizes the costs required to apply a skill, and the $\sum$ values on the bars represent the total skill costs at each workstation. We can notice, based on the used skills, that the $w_{4}-w_{7}$ workstations are similar, which means the activities between these stations can be reallocated without causing additional cost by training. Furthermore, there is a high correlation between skills and types of equipment so that it would require no additional tool or resource. Considering these, an update or redesign of activity assignments among workstations could reduce the cost of the assembly process.

In the following, we analyzed the $p_{1}$ product where only the base module $\left(m_{0}\right)$ is assembled because this is the most relevant one (see Figure 16). The line balancing has been analyzed to perform further investigations. Figure 18(a) shows the current line balancing in the case of the $p_{1}$ product. It can be noticed that this is not a well-balanced production process. However, we need to follow the procedure of the applied conveyor line. In an open-paced conveyor, the start and the ending stations have more flexibility than the middle ones. Based on this, the operators in the middle stages $\left(w_{3}-w_{8}\right)$ are usually planned for lower capacity. Apart from this, we can also highlight that 


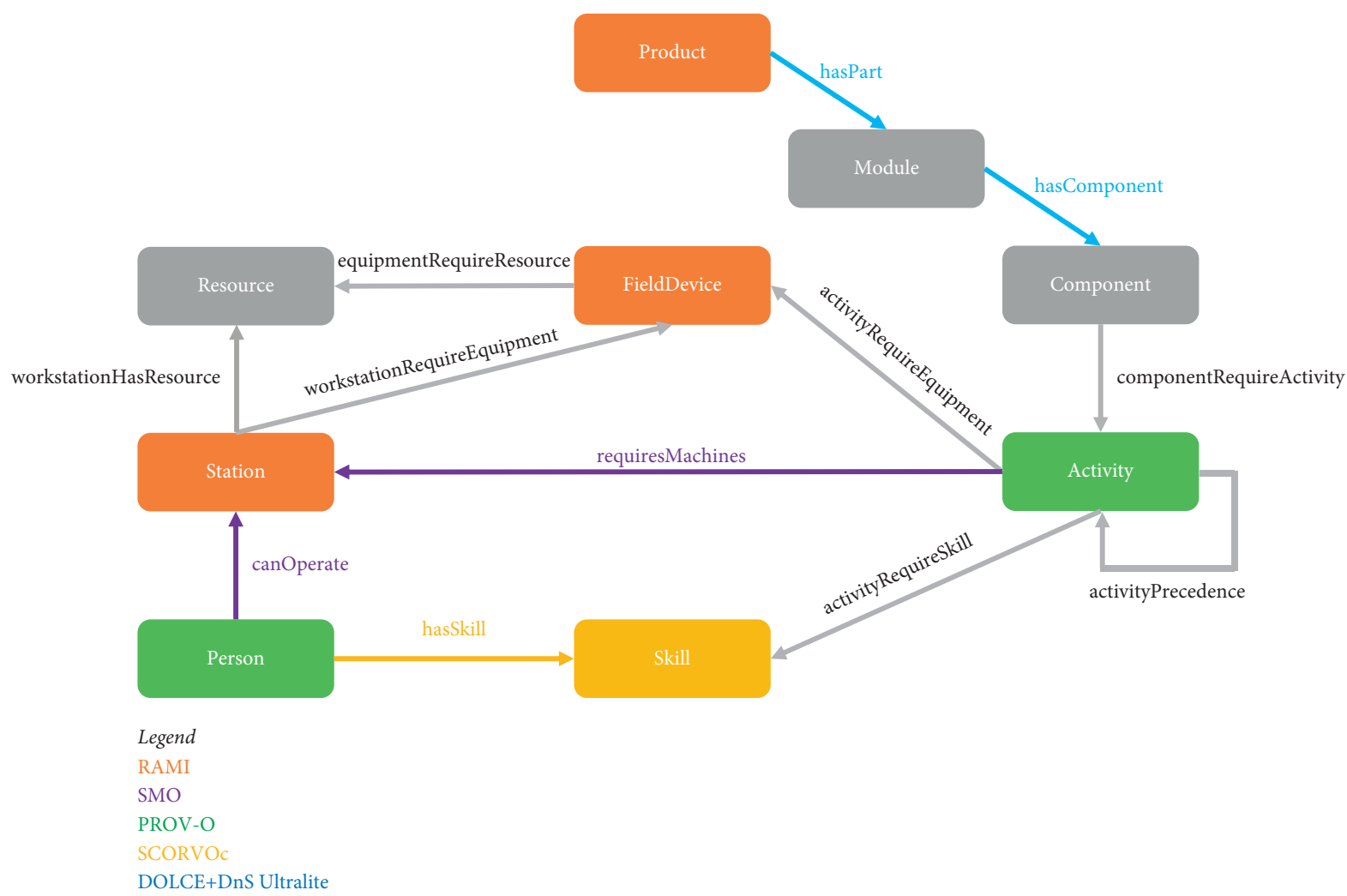

FIgURE 14: The ontology model of a wire harness assembly line based on the applied vocabulary and namespace elements.

TABLE 3: List of the applied object and data properties.

\begin{tabular}{lc}
\hline Object properties & Data properties \\
\hline hasPart & activityTime \\
hasComponent & activityType \\
componentRequireActivity & activityTypeName \\
activityPrecedence & activityTypeRemark \\
canOperate & activityTypeUnit \\
requiresMachine & additionalTime \\
activityRequireSkill & componentType \\
equipmentRequireResource & zoneOfAssembly \\
activityRequireEquipment & equipmentName\&equipmentCost \\
workstationHasResource & resourceName\&resourceCost \\
hasSkill & skillName\&skillCost \\
workstationRequireEquipment & moduleName \\
\hline
\end{tabular}

the differences are significant, nearly a minute between these stations, which is an opportunity to make further analyses to discover the potential of merging these workstations.

In the next approach, we analyze the skill, equipment, and workstation assignment in assembly activities using MuxViz. Figure 19 shows the ontology-based multilayer visualisation, which contains three different layers, representing the connectivity of the 653 assembly activities to other classes of the ontology as skills, pieces of equipment, and workstations. Unique colours denote the core nodes of the network as five skills (green), five pieces of equipment (blue), and ten workstations (red). Additional information is presented by internal edges within these three layers, connecting the core nodes and representing assignments as workstation-skill, skill-equipment, and workstationequipment.

The activity-workstation layer is investigated in more detail to uncover the possibility of merging activities within $w_{3}-w_{8}$ stations. Figure 20 presents the discovered communities based on multilayer connectivity, where workstations are classified into five communities $\left(\varphi_{1}-\varphi_{5}\right)$, which are listed in Table 4 . We can conclude that the identified, highly related workstations are included in the same community based on several attributes. The multilayer analyses confirmed that merging these stations would be beneficial.

Figure 18(b) shows the result of line balancing after reallocation assembly activities related to $w_{3}-w_{6}$ stations and eliminating the $w_{7}$ station from the line. Based on the presented advanced analytics, we could eliminate one of the workstations (and one operator) from the production line. It is possible to redesign the conveyor line with 9 stations instead of 10. Although there are still gaps among stations, this is more efficient as the starting point. We need to highlight that we are focusing on one type of product only, and also, the open-paced conveyor has a special line balancing rule, as mentioned above. However, the SPARQLbased data queries and multilayer analyses can make more efficient the discovering of communities and critical elements of the production system. These show the possibilities for process engineers to solve the line balancing problem considering all production parameters. 


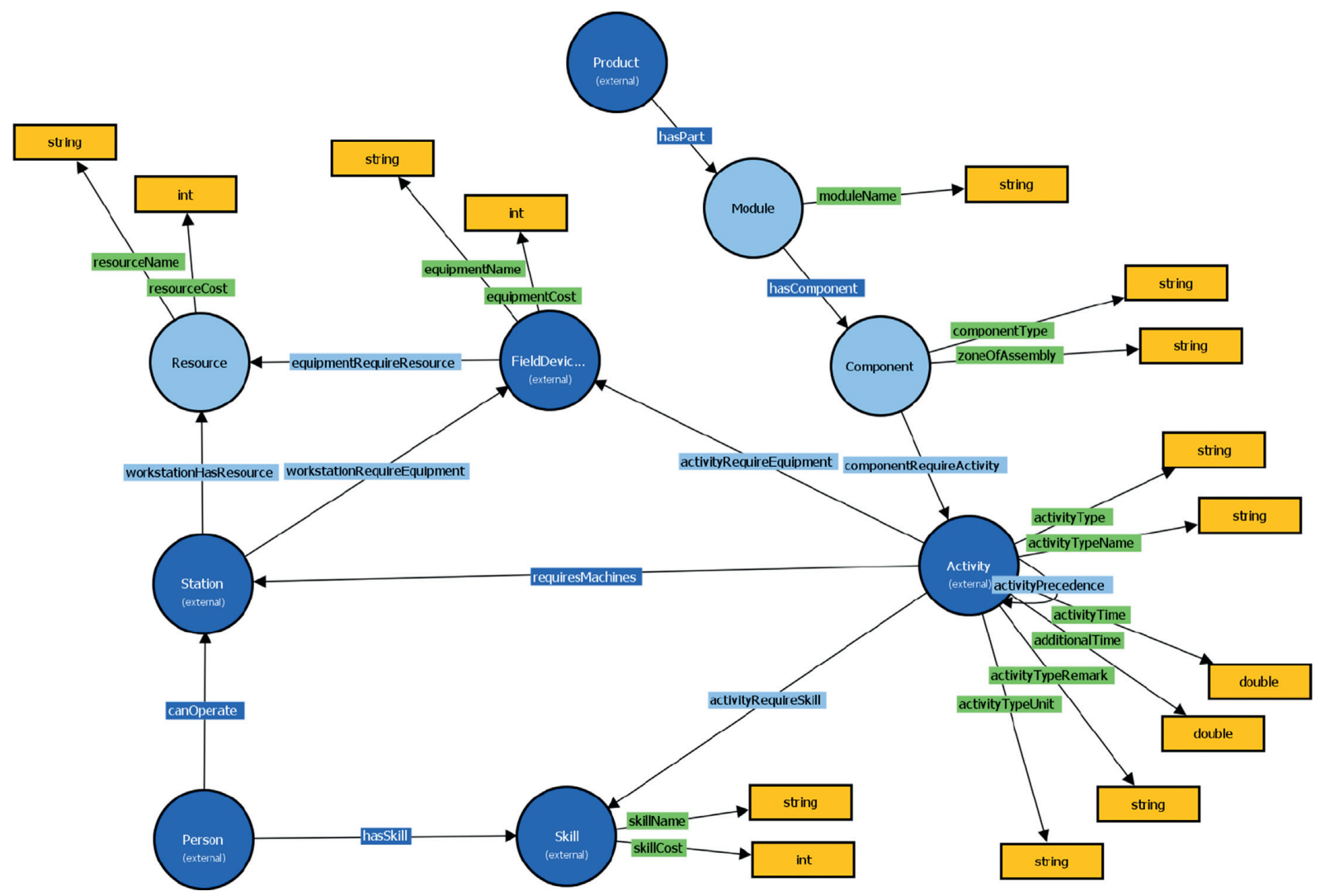

FIgURE 15: The VOWL view of the created ontology of the wire harness manufacturing process.

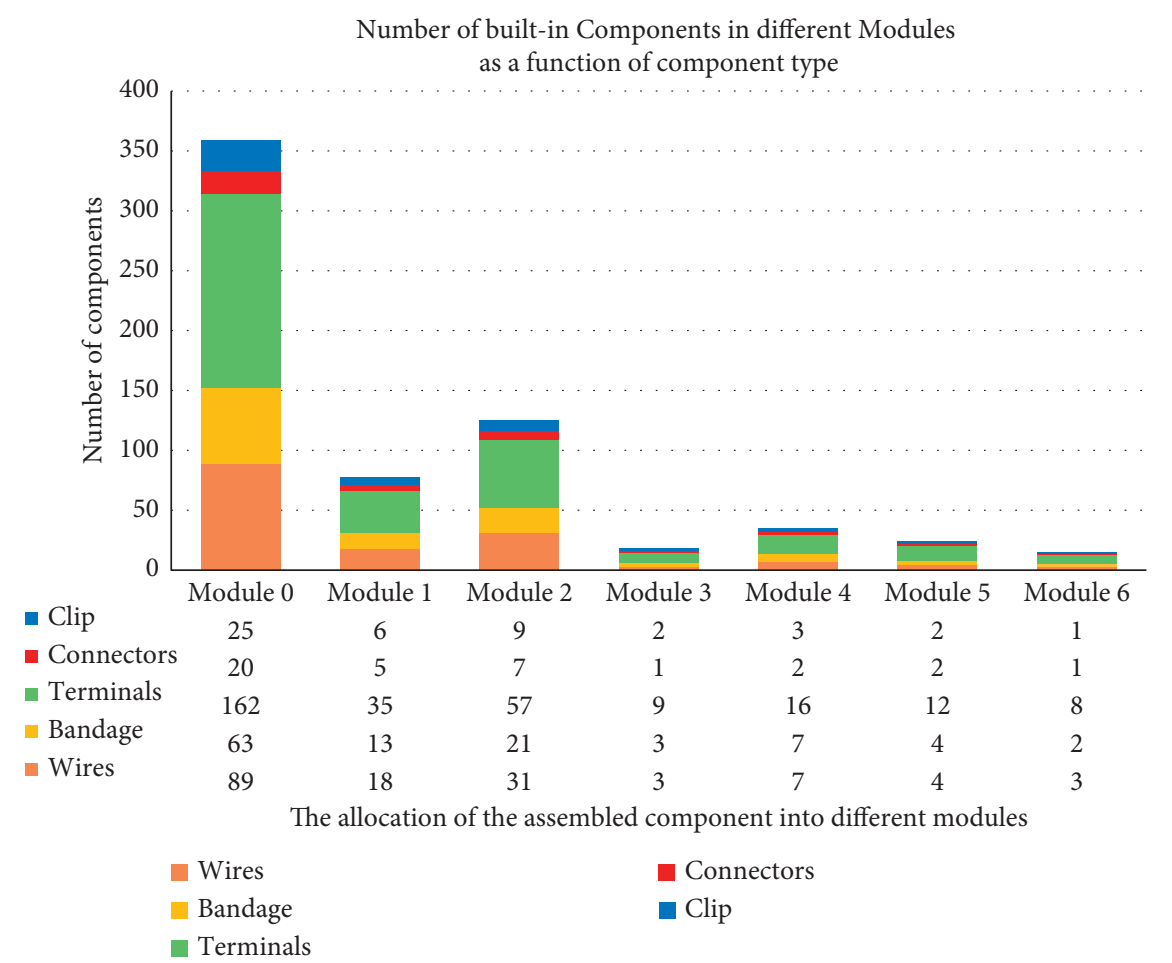

FIGURE 16: Results of the SPARQL query regarding built-in components in different modules. 


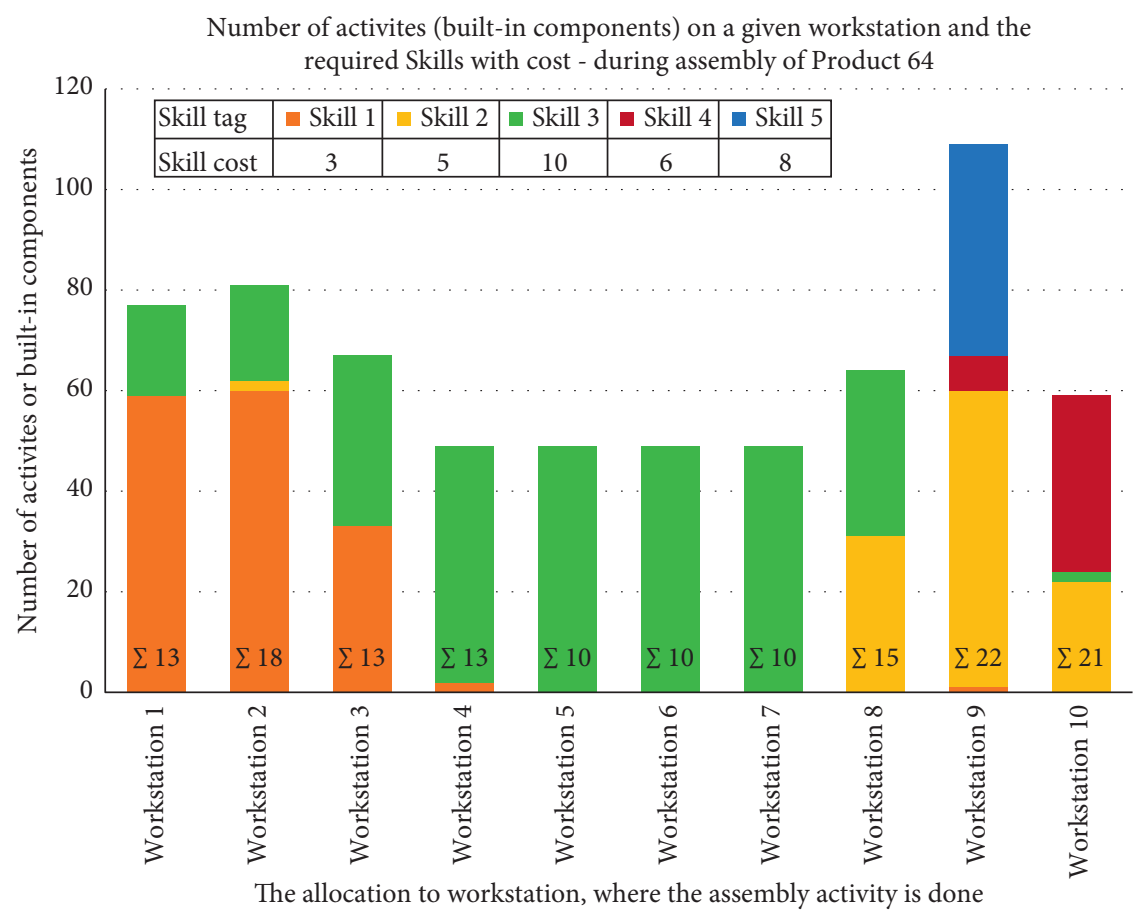

FIGURE 17: Results of the SPARQL query regarding workstation allocation and skill usage during the assembly of product 64 .

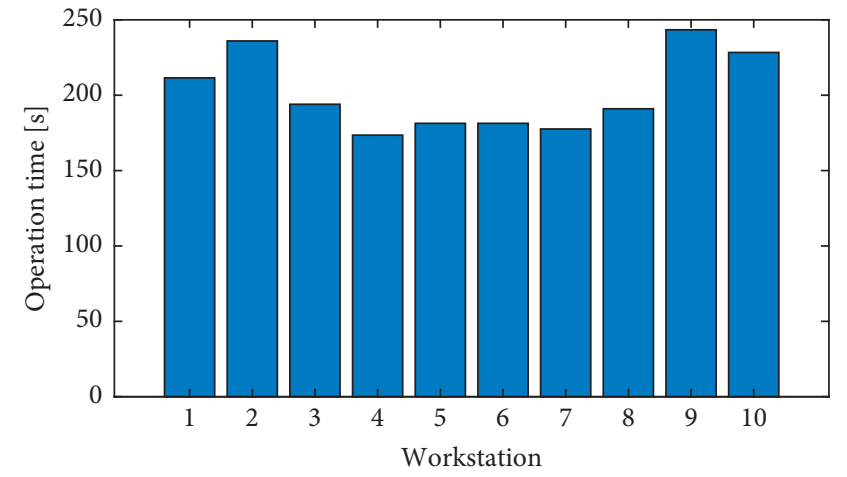

(a)

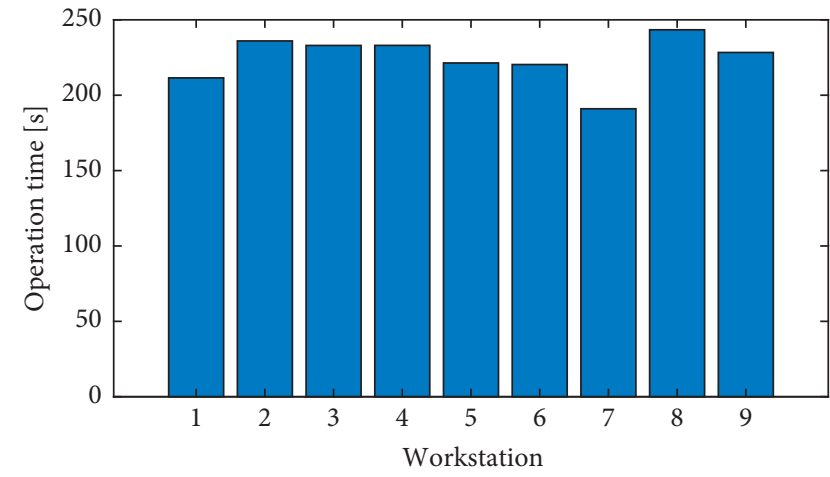

(b)

FIgURE 18: The evolution of manufacturing time during assembly of the $p_{1}$ product before and after line balancing. (a) The original case. (b) After line balancing.

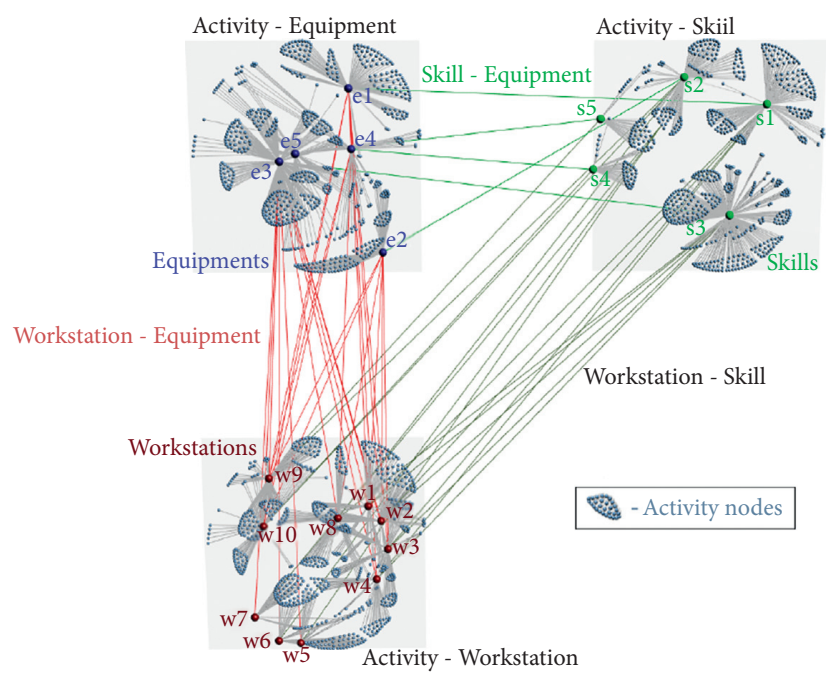

Figure 19: Multilayer visualisation of wire harness manufacturing data. 


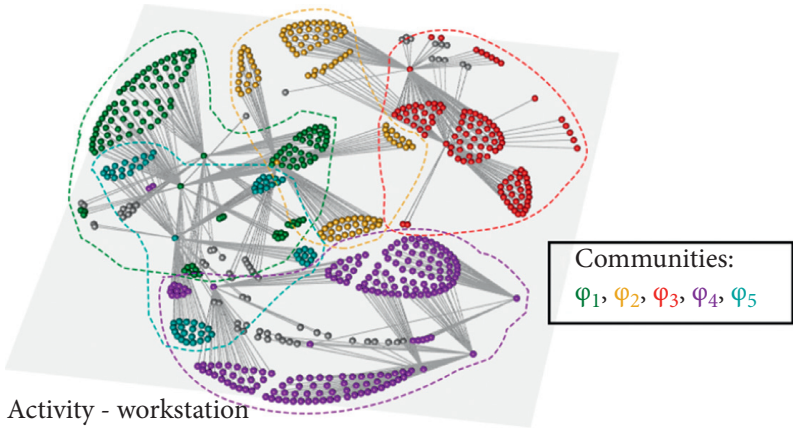

Figure 20: Activity-workstation layer and the identified communities.

TABle 4: The distribution of workstations into five different communities.

\begin{tabular}{lccc}
\hline Workstation & Community & Workstation & Community \\
\hline$w_{1}$ & $\varphi_{2}$ & $w_{6}$ & $\varphi_{4}$ \\
$w_{2}$ & $\varphi_{2}$ & $w_{7}$ & $\varphi_{4}$ \\
$w_{3}$ & $\varphi_{3}$ & $w_{8}$ & $\varphi_{5}$ \\
$w_{4}$ & $\varphi_{4}$ & $w_{9}$ & $\varphi_{1}$ \\
$w_{5}$ & $\varphi_{4}$ & $w_{10}$ & $\varphi_{1}$ \\
\hline
\end{tabular}

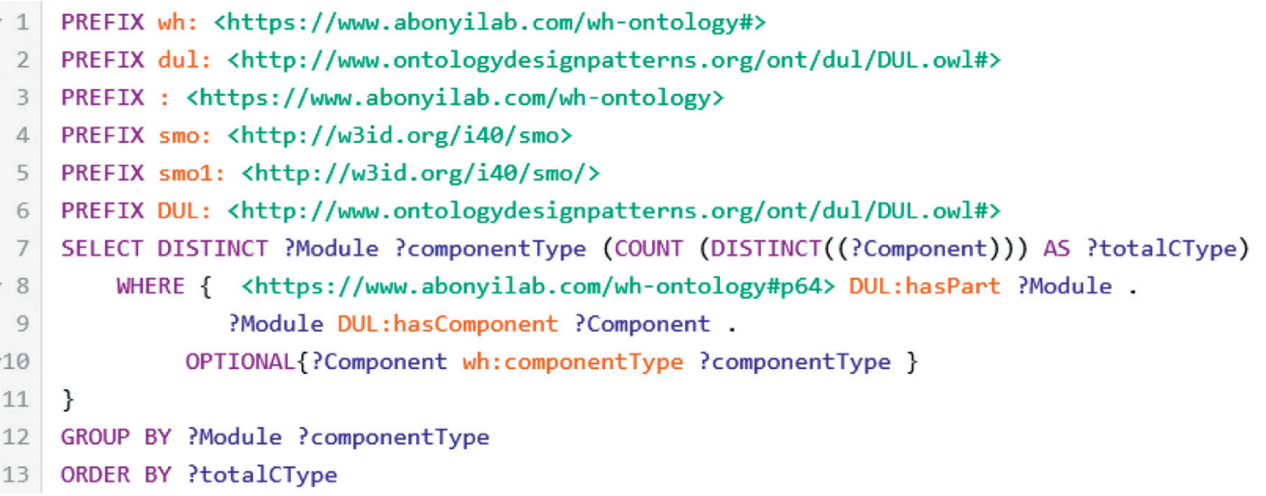

FIGURE 21: SPARQL query-module-component.

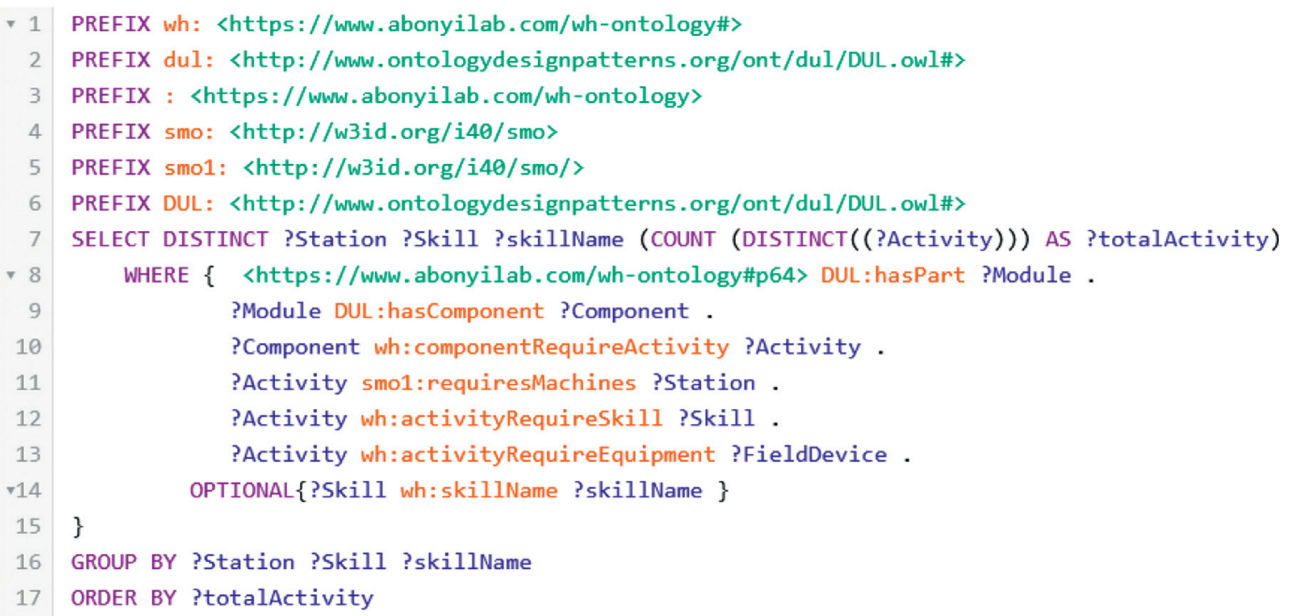

FIGURE 22: SPARQL query-workstation-skill. 
TABLe 5: Details of the activities during the wire harness assembly.

\begin{tabular}{|c|c|c|c|c|}
\hline ID & Activity & Remark & Unit & Time (s) \\
\hline $\bar{t} t_{1}$ & Point-to-point wiring on chassis & Direct wiring & Per wire & 1.5 \\
\hline$t_{2}$ & Laying in the U-channel & & & 2.0 \\
\hline$t_{3}$ & Laying flat cable & & & 4.0 \\
\hline$t_{4}$ & & & Base time & 2.5 \\
\hline$\iota_{4}$ & Laying wires onto the harness jig & Laying flat cable & Per wire & 5.0 \\
\hline$t_{5}$ & Laying the cable connector (one end) onto the harness jig & To the same breakout & Base time & 3.2 \\
\hline & & & Per wire & 2.3 \\
\hline$t_{6}$ & Spot-tying onto the cable and cutting it with a pair of scissors & & & 3.3 \\
\hline$t_{7}$ & Lacing activity & & & 1.25 \\
\hline$t_{8}$ & Taping activity & & & 1.0 \\
\hline$t_{9}$ & Inserting into the tube or sleeve & & & 1.5 \\
\hline$t_{10}$ & Attachment of the wire terminal & Terminal-block fastening (fork lug) & & 6.5 \\
\hline$t_{11}$ & Screw fastening of the terminal & & & 7.55 \\
\hline$t_{12}$ & Screw-and-nut fastening of the terminal & & & 12.35 \\
\hline$t_{13}$ & Circular connector & Installation only & & 5.65 \\
\hline$t_{14}$ & Rectangular connector & Latch or snap-on & & 11.0 \\
\hline$t_{15}$ & Clip installation & & & 4.0 \\
\hline
\end{tabular}

\section{Conclusion}

In this paper, we proposed an ontology development methodology with a wire harness assembly-based benchmark to describe the ontologies of manufacturing processes as well as provide an overview and guidance on development for others. The ontological modelling of a manufacturing process and the performance of advanced manufacturing analytics on the formalised data is a very complex procedure. Below, the lessons which have been learned during this research are summarized to provide a conclusion and takeaway message for the reader:

(i) The integration of ISA and IEC standards is of great importance in semantic model-based system development

(ii) In-depth study of Open Vocabularies can facilitate ontological and semantic modelling

(iii) There is a need to use and develop industry-specific ontologies and knowledge graphs

(iv) Multilayer graphs are suitable tools for analysing data stored in ontologies

(v) Network and data science can support the analysis of complex systems represented by ontologies

(vi) The multilayer-network-based analysis of ontologies supports production management

In the future, we plan to extend our research using other case studies as well and start to develop our very own Ontology-Based Data Access (OBDA) system. We will consider performing time-stamped data queries on live, ontological structured data.

\section{Appendix}

\section{A. SPARQL Queries}

Figure 21 shows the SPARQL query to get the modulecomponent results for Figures 16 and 22 describes the query for workstation-skill analyses regarding the workstation allocation and skill usage (see Figure 17).

\section{B. Wire Harness Assembly Activity Times}

The types of activities and the related activity times according to wire harness assembly practice [93] are summarized in Table 5. The activity times are calculated using a direct proportionality approach, e.g., when an operator is laying four wires over one foot, proportionally to the parameter $t_{4}$, the activity time will be $1 \times 6.9 \mathrm{~s}+4 \times 4.2 \mathrm{~s}=23.7 \mathrm{~s}$.

\section{Data Availability}

The wire harness assembly data used to support the findings of this study are deposited in the GitHub repository (https:// github.com/abonyilab/wire_harness_assembly).

\section{Conflicts of Interest}

The authors declare no conflicts of interest.

\section{Acknowledgments}

This work was supported by the TKP2020-NKA-10 Project financed under the 2020-4.1.1-TKP2020 Thematic Excellence Programme by the National Research, Development and Innovation Fund of Hungary and 2019-1.1.1-PIACIKFI-2019-00312 Project (Mobilized Collaborative RobotBased Development of a Modular Industry 4.0 Production System with Quality Management Functions).

\section{References}

[1] S. Jaskó, A. Skrop, T. Holczinger, T. Chován, and J. Abonyi, "Development of manufacturing execution systems in accordance with Industry 4.0 requirements: a review of standard- and ontology-based methodologies and tools," Computers in Industry, vol. 123, Article ID 103300, 2020.

[2] Z. Ming, G. Sharma, J. K. Allen, and F. Mistree, "An ontology for representing knowledge of decision interactions in 
decision-based design," Computers in Industry, vol. 114, Article ID 103145, 2020.

[3] C. Kardos, G. Popovics, B. Kádár, and L. Monostori, "Methodology and data-structure for a uniform system's specification in simulation projects," Procedia CIRP, vol. 7, pp. 455-460, 2013.

[4] W. Van Der Aalst, "Process mining," Communications of the ACM, vol. 55 , no. 8, pp. 76-83, 2012.

[5] I. Yürek, D. Birant, Ö. C. Yurek, and K. U. Birant, "Interactive process miner: a new approach for process mining," Turkish Journal of Electrical Engineering and Computer Sciences, vol. 26, no. 3, pp. 1314-1328, 2018.

[6] L. González and A. Hogan, "Modelling dynamics in semantic web knowledge graphs with formal concept analysis," in Proceedings of the 2018 World Wide Web Conference, pp. 1175-1184, Monterey, CA, USA, 2018.

[7] A. Maedche and S. Staab, "Ontology learning for the semantic web," IEEE Intelligent Systems, vol. 16, no. 2, pp. 72-79, 2001.

[8] R. Angles, M. Arenas, P. Barceló, A. Hogan, J. Reutter, and D. Vrgoč, "Foundations of modern query languages for graph databases," ACM Computing Surveys, vol. 50, no. 5, pp. 1-40, 2017.

[9] N. Sahlab, S. Kamm, T. Müller, N. Jazdi, and M. Weyrich, "Knowledge graphs as enhancers of intelligent digital twins," in Proceedings of the 2021 4th IEEE International Conference on Industrial Cyber-Physical Systems (ICPS), pp. 19-24, IEEE, Victoria, BC, Canada, May 2021.

[10] G. Stefansson, "Business-to-business data sharing: a source for integration of supply chains," International Journal of Production Economics, vol. 75, no. 1-2, pp. 135-146, 2002.

[11] O. A. Amodu and M. Othman, "Machine-to-machine communication: an overview of opportunities," Computer Networks, vol. 145, pp. 255-276, 2018.

[12] G. F. Schneider, H. Wicaksono, and J. Ovtcharova, "Virtual engineering of cyber-physical automation systems: the case of control logic," Advanced Engineering Informatics, vol. 39, pp. 127-143, 2019.

[13] L. Yang, K. Cormican, and M. Yu, "Ontology-based systems engineering: a state-of-the-art review," Computers in Industry, vol. 111, pp. 148-171, 2019.

[14] T. Liebig, A. Maisenbacher, M. Opitz, J. R. Seyler, G. Sudra, and J. Wissmann, Building a Knowledge Graph for Products and Solutions in the Automation Industry. Computer Science, Festo, Germany, 2019.

[15] J. Pujara, H. Miao, L. Getoor, and W. Cohen, "Knowledge graph identification," in Proceedings of the International Semantic Web Conference, pp. 542-557, Springer, Sydney, Australia, 2013.

[16] E. Wallace, D. Kiritsis, B. Smith, C. Will, and B. Kulvatunyou, "The industrial ontologies foundry proofof-concept project," in Proceedings of the IFIP International Conference on Advances in Production Management Systems, pp. 402-409, Springer, Seoul, August 2018.

[17] M. Karray, N. Otte, R. Rai et al., "The industrial ontologies foundry (IOF) perspectives," in Proceedings of the Industrial Ontology Foundry (IOF)-Achieving Data Interoperability Workshop, Tarbes, France, November 2021.

[18] S. Borgo, F. Compagno, N. Guarino, C. Masolo, and E. M. Sanfilippo, "An overview of some ontological challenges in engineering maintenance," Domain Ontologies for Research Data Management in Industry Commons of Materials and Manufacturing, vol. 1, 2021.
[19] C. Kardos, A. Kovács, and J. Váncza, "A constraint model for assembly planning," Journal of Manufacturing Systems, vol. 54, pp. 196-203, 2020.

[20] M. Fechter, C. Seeber, and S. Chen, "Integrated process planning and resource allocation for collaborative robot workplace design," Procedia CIRP, vol. 72, pp. 39-44, 2018.

[21] Gartner and K. Panetta, 5 Trends Drive the Gartner Hype Cycle for Emerging Technologies, https://www.gartner.com. https://www.gartner.com/smarterwithgartner/5-trendsdrive-the-gartner-hype-cycle-for-emerging-technologies2020/, 2020.

[22] Gartner and K. Panetta, Gartner Top 10 Strategic Technology Trends for 2020, https://www.gartner.com". https://www. gartner.com/smarterwithgartner/gartner-top-10-strategictechnology-trends-for-2020/, 2019.

[23] M. Hankel and B. Rexroth, The Reference Architectural Model Industrie 4.0 (Rami 4.0), p. 410, ZVEI, Frankfurt, Germany, 2015.

[24] P. Leitao, A. W. Colombo, and F. J. Restivo, "ADACOR: a collaborative production automation and control architecture," IEEE Intelligent Systems, vol. 20, no. 1, pp. 58-66, 2005.

[25] T. Ruppert, G. Honti, and J. Abonyi, "Multilayer networkbased production flow analysis," Complexity, vol. 1, 2018.

[26] L. Nagy, T. Ruppert, and J. Abonyi, "Analytic hierarchy process and multilayer network-based method for assembly line balancing," Applied Sciences, vol. 10, no. 11, p. 3932, 2020.

[27] G. Honti, G. Dörgő, and J. Abonyi, "Network analysis dataset of system dynamics models," Data in Brief, vol. 27, Article ID 104723, 2019.

[28] M. Hannah, C. Leiva, and D. Noller, "The importance of standards in smart manufacturing," MESA International White Paper, vol. 58, 2018.

[29] H. O. Unver, "An ISA-95-based manufacturing intelligence system in support of lean initiatives," International Journal of Advanced Manufacturing Technology, vol. 65, no. 5-8, pp. 853-866, 2013.

[30] D. He, A. Lobov, and J. M. Lastra, "ISA-95 tool for enterprise modeling," in Proceedings of the ICONS 2012: The Seventh International Conference on Systems, pp. 83-87, Lisbon, Portugal, November 2012.

[31] H. Panetto, S. Baïna, and G. Morel, "Mapping the IEC 62264 models onto the Zachman framework for analysing products information traceability: a case study," Journal of Intelligent Manufacturing, vol. 18, no. 6, pp. 679-698, 2007.

[32] G. Booch, The Unified Modeling Language User Guide, Pearson Education India, London, UK, 2005.

[33] S. W. Lin, B. Murphy, E. Clauer et al., Architecture Alignment and Interoperability: An Industrial Internet Consortium and Platform Industrie 4.0 Joint Whitepaper, Industrial Internet Consortium, Boston, MA, USA, 2017.

[34] H. Xu, W. Yu, D. Griffith, and N. Golmie, "A survey on industrial Internet of Things: a cyber-physical systems perspective," IEEE Access, vol. 6, pp. 78238-78259, 2018.

[35] K. Schweichhart, Reference Architectural Model Industrie 4.0 (Rami 4.0), p. 40, 2016 An Introduction Available online: https://www.plattform-i40.de/IP/Navigation/DE/Home/ home.html.

[36] I. Horrocks, B. Parsia, P. Patel-Schneider, and J. Hendler, "Semantic web architecture: Stack or two towers?" in Proceedings of the International Workshop on Principles and Practice of Semantic Web Reasoning, pp. 37-41, Springer, Dagstuhl Castle, Germany, 2005. 
[37] M. Kifer, J. De Bruijn, H. Boley, and D. Fensel, "A realistic architecture for the semantic web," in Proceedings of the International Workshop on Rules and Rule Markup Languages for the Semantic Web, pp. 17-29, Springer, Galway, Ireland, 2005.

[38] I. Szilagyi and P. Wira, "Ontologies and semantic web for the Internet of things-a survey," in Proceedings of the IECON 2016-42nd Annual Conference of the IEEE Industrial Electronics Society, pp. 6949-6954, IEEE, Florence, Italy, October 2016.

[39] D. Brickley, R. V. Guha, and A. Layman, Resource Description Framework (RDF) Schema Specification, W3C, Cambridge, MA, USA, 1999.

[40] D. L. McGuinness and F. Van Harmelen, "OWL web ontology language overview," W3C Recommendation, vol. 10, no. 10, 2004.

[41] A. Brodt, D. Nicklas, and B. Mitschang, "Deep integration of spatial query processing into native RDF triple stores," in Proceedings of the 18th SIGSPATIAL International Conference on Advances in Geographic Information Systems, pp. 33-42, San Jose, CA, USA, November 2010.

[42] P. Zangeneh and B. McCabe, "Ontology-based knowledge representation for industrial megaprojects analytics using linked data and the semantic web," Advanced Engineering Informatics, vol. 46, Article ID 101164, 2020.

[43] P. Hitzler, M. Krötzsch, B. Parsia, and S. Rudolph, "OWL 2 web ontology language primer," W3C Recommendation, vol. 27, no. 1, p. 123, 2009.

[44] J. Pérez, M. Arenas, and C. Gutierrez, "Semantics and complexity of SPARQL," ACM Transactions on Database Systems, vol. 34, no. 3, pp. 1-45, 2009.

[45] O. Meski, F. Belkadi, F. Laroche, and B. Furet, "Towards a knowledge-based framework for digital chain monitoring within the industry 4.0 paradigm," Procedia CIRP, vol. 84, pp. 118-123, 2019.

[46] S. R. Bader, I. Grangel-González, M. Tasnim, and S. Lohmann, "Structuring the industry 4.0 landscape," in Proceedings of the 2019 24th IEEE International Conference on Emerging Technologies and Factory Automation (ETFA), pp. 224-231, IEEE, Zaragoza, Spain, 2019.

[47] I. Grangel-González, P. Baptista, L. Halilaj et al., "The industry 4.0 standards landscape from a semantic integration perspective," in Proceedings of the 2017 22nd IEEE International Conference on Emerging Technologies and Factory Automation (ETFA), pp. 1-8, IEEE, 2017.

[48] A. Löcklin, H. Vietz, D. White, T. Ruppert, N. Jazdi, and M. Weyrich, "Data administration shell for data-sciencedriven development," Procedia CIRP, vol. 100, pp. 115-120, 2021.

[49] I. Grangel-González, L. Halilaj, G. Coskun, S. Auer, D. Collarana, and M. Hoffmeister, "Towards a semantic administrative shell for industry 4.0 components," in Proceedings of the 2016 IEEE Tenth International Conference on Semantic Computing (ICSC), pp. 230-237, IEEE, Laguna Hills, California, February 2016.

[50] R. Drath, A. Luder, J. Peschke, and L. Hundt, "AutomationML-the glue for seamless automation engineering," in Proceedings of the 2008 IEEE International Conference on Emerging Technologies and Factory Automation, pp. 616-623, IEEE, Hamburg, Germany, 2008.

[51] O. Kovalenko, I. Grangel-González, M. Sabou et al., "AutomationML ontology: modeling cyber-physical systems for industry 4.0," IOS Press Journal, vol. 1, 2018.
[52] K. Janowicz, A. Haller, S. J. D. Cox, D. Le Phuoc, and M. Lefrançois, "SOSA: a lightweight ontology for sensors, observations, samples, and actuators," Journal of Web Semantics, vol. 56, pp. 1-10, 2019.

[53] E. Järvenpää, N. Siltala, O. Hylli, and M. Lanz, “The development of an ontology for describing the capabilities of manufacturing resources," Journal of Intelligent Manufacturing, vol. 30, no. 2, pp. 959-978, 2019.

[54] N. Seydoux, K. Drira, N. Hernandez, and T. Monteil, "IoT-O, a core-domain IoT ontology to represent connected devices networks," in Proceedings of the European Knowledge Acquisition Workshop, pp. 561-576, Springer, Bologna, Italy, 2016.

[55] R. Arp, B. Smith, and A. D. Spear, Building Ontologies with Basic Formal Ontology, Mit Press, Cambridge, BA, USA, 2015.

[56] J. Wang, J. Liu, and Y. Zhong, "A novel ant colony algorithm for assembly sequence planning," International Journal of Advanced Manufacturing Technology, vol. 25, no. 11-12, pp. 1137-1143, 2005.

[57] F. Ameri and D. Dutta, "An upper ontology for manufacturing service description," in Proceedings of the International Design Engineering Technical Conferences and Computers and Information in Engineering Conference, pp. 651-661, Philadelphia, USA, September 2006.

[58] A. Gangemi, N. Guarino, C. Masolo, A. Oltramari, and L. Schneider, "Sweetening ontologies with DOLCE," in Proceedings of the International Conference on Knowledge Engineering and Knowledge Management, pp. 166-181, Springer, Sigüenza, Spain, 2002.

[59] A. Scherp, T. Franz, C. Saathoff, and S. Staab, "F-a model of events based on the foundational ontology dolce + DnS ultralight," in Proceedings of the Fifth International Conference on Knowledge Capture, pp. 137-144, Redondo Beach, California, USA, September 2009.

[60] B. Wally, C. Huemer, A. Mazak, and M. Wimmer, "IEC 62264-2 for AutomationML," in Proceedings of the 5th AutomationML User Conference, pp. 1-7, Gothenburg, Sweden, October 2018.

[61] K. Kotis and A. Katasonov, "An IoT-ontology for the representation of interconnected, clustered and aligned smart entities," Technical Report, VTT Technical Research Center, VTT Technical Research Center, Finland, 2012.

[62] R. Batres, M. West, D. Leal et al., "An upper ontology based on ISO 15926," Computers \& Chemical Engineering, vol. 31, no. 5-6, pp. 519-534, 2007.

[63] A. Iliadis, "The tower of babel problem: making data make sense with basic formal ontology," Online Information Review, vol. 43, 2019.

[64] For Standardization IO, "ISO/IEC 21838-2 Information technology: top-level ontologies (TLO) - part 2: basic formal ontology (BFO)," ISO, International Organization for Standardization, London, UK, 2021, https://www.iso.org/ standard/74572.html

[65] Y. Zhong, C. Jiang, Y. Qin, G. Yang, M. Huang, and X. Luo, "Automatically generating assembly sequences with an ontology-based approach," Assembly Automation, vol. 40, no. 2, pp. 319-334, 2019.

[66] F. Roda and E. Musulin, "An ontology-based framework to support intelligent data analysis of sensor measurements," Expert Systems with Applications, vol. 41, no. 17, pp. 79147926, 2014.

[67] V. Presutti and A. Gangemi, "Content ontology design patterns as practical building blocks for web ontologies," in 
Proceedings of the International Conference on Conceptual Modeling, pp. 128-141, Springer, Barcelona, Spain, October 2008.

[68] Z. Huang, C. Jowers, A. Dehghan-Manshadi, and M. S. Dargusch, "Smart manufacturing and DVSM based on an Ontological approach," Computers in Industry, vol. 117, Article ID 103189, 2020.

[69] I. Harjunkoski, M. Hollender, R. Bauer, J. Doppelhamer, S. Subbiah, and W. Schmidt, "An easy-to-use and flexible scheduling component," Control and Productivity, vol. 3, 2017.

[70] S. Ahmadi, "Ontology validation of manufacturing execution systems through the analysis of semantic descriptions," Master's thesis, Tampere University, Tampere, Finland, 2018.

[71] I. Grangel-González, L. Halilaj, M.-E. Vidal et al., "Knowledge graphs for semantically integrating cyber-physical systems," in Proceedings of the International Conference on Database and Expert Systems Applications, pp. 184-199, Springer, Regensburg, Germany, 2018.

[72] G. N. Schroeder, C. Steinmetz, C. E. Pereira, and D. B. Espindola, "Digital twin data modeling with automation $\mathrm{ml}$ and a communication methodology for data exchange," IFAC-PapersOnLine, vol. 49, no. 30, pp. 12-17, 2016.

[73] B. Wally, L. Lang, R. Włodarski et al., "Generating structured AutomationML models from IEC 62264 information," in Proceedings of the 5th AutomationML PlugFest 2019, Hamburg, Germany, 2019.

[74] A. Argyrou, C. Giannoulis, N. Papakostas, and G. Chryssolouris, "A uniform data model for representing symbiotic assembly stations," Procedia CIRP, vol. 44, pp. 85-90, 2016.

[75] R. Müller, L. Hörauf, M. Vette, and C. Speicher, "Planning and developing cyber-physical assembly systems by connecting virtual and real worlds," Procedia Cirp, vol. 52, pp. 35-40, 2016.

[76] R. Kretschmer, A. Pfouga, S. Rulhoff, and J. Stjepandić, "Knowledge-based design for assembly in agile manufacturing by using Data Mining methods," Advanced Engineering Informatics, vol. 33, pp. 285-299, 2017.

[77] M. Ringsquandl, S. Lamparter, S. Brandt, T. Hubauer, and R. Lepratti, "Semantic-guided feature selection for industrial automation systems," in Proceedings of the International Semantic Web Conference, pp. 225-240, Springer, Bethlehem, PA, USA, 2015.

[78] E. Kharlamov, E. Jiménez-Ruiz, D. Zheleznyakov et al., "Optique: towards OBDA systems for industry," in Proceedings of the Extended Semantic Web Conference, pp. 125-140, Springer, Montpellier, France, 2013.

[79] E. Kharlamov, N. Solomakhina, Ö. L. Özçep et al., "How semantic technologies can enhance data access at siemens energy," in Proceedings of the International Semantic Web Conference, pp. 601-619, Springer, Riva del Garda, Italy, 2014.

[80] E. Kharlamov, B. C. Grau, E. Jiménez-Ruiz et al., "Capturing industrial information models with ontologies and constraints," in Proceedings of the International Semantic Web Conference, pp. 325-343, Springer, Kobe, Japan, 2016.

[81] M. Ringsquandl, S. Lamparter, and R. Lepratti, "Graph-based predictions and recommendations in flexible manufacturing systems," in Proceedings of the IECON 2016-42nd Annual
Conference of the IEEE Industrial Electronics Society, pp. 6937-6942, IEEE, Florence, Italy, October 2016.

[82] J. Hayes and C. Gutierrez, "Bipartite graphs as intermediate model for RDF," in Proceedings of the International Semantic Web Conference, pp. 47-61, Springer, Hiroshima, Japan, 2004.

[83] G. Honti and J. Abonyi, "Frequent item set mining and multi-layer network-based analysis of RDF databases," Mathematics, vol. 9, no. 4, p. 450, 2021.

[84] Stanford University, Protégé-A Free, Open-Source Ontology Editor and Framework for Building Intelligent Systems, Stanford University, Stanford, CA, USA, 2020, https:// protege.stanford.edu/.

[85] Ontotext, "GraphDB - an enterprise ready semantic graph database, compliant with W3C stan-dards \lenleadertwodots ontotext," 2020, https://graphdb.ontotext.com/.

[86] M. D. Domenico, "MuxViz-the multilayer analysis and visualization platform \lenleadertwodots," 2020, https:// muxviz.net/.

[87] N. F. Noy, M. Crubézy, R. W. Fergerson et al., "Protégé-2000: an open-source ontology-development and knowledge-acquisition environment," in Proceedings of the AMIA Annual Symposium, p. 953, Washington, DC, USA, February 2003.

[88] J. H. Gennari, M. A. Musen, R. W. Fergerson et al., "The evolution of Protégé: an environment for knowledge-based systems development," International Journal of HumanComputer Studies, vol. 58, no. 1, pp. 89-123, 2003.

[89] P. Y. Vandenbussche, G. A. Atemezing, M. Poveda-Villalón, and B. Vatant, "Linked Open Vocabularies (LOV): a gateway to reusable semantic vocabularies on the Web," Semantic Web, vol. 8, no. 3, pp. 437-452, 2017.

[90] M. De Domenico, M. A. Porter, and A. Arenas, "MuxViz: a tool for multilayer analysis and visualization of networks," Journal of Complex Networks, vol. 3, no. 2, pp. 159-176, 2015.

[91] H. Cheng, P. Zeng, L. Xue, Z. Shi, P. Wang, and H. Yu, "Manufacturing ontology development based on Industry 4.0 demonstration production line," in Proceedings of the 2016 Third International Conference on Trustworthy Systems and their Applications (TSA), pp. 42-47, IEEE, Wuhan, Hubei, September 2016.

[92] S. Lohmann, S. Negru, and D. Bold, "The ProtégéVOWL plugin: ontology visualization for everyone," in Proceedings of the European Semantic Web Conference, pp. 395-400, Springer, Crete, Greece, 2014.

[93] N. S. Ong and G. Boothroyd, "Assembly times for electrical connections and wire harnesses," International Journal of Advanced Manufacturing Technology, vol. 6, no. 2, pp. 155179, 1991.

[94] T. Ruppert and J. Abonyi, "Software sensor for activity-time monitoring and fault detection in production lines," Sensors, vol. 18, no. 7, p. 2346, 2018.

[95] N. S. Ong, "Activity-based cost tables to support wire harness design," International Journal of Production Economics, vol. 29, no. 3, pp. 271-289, 1993.

[96] N. P. Irlan Grangel, "RAMI vocabulary," 2015, https://i40. semantic-interoperability.org, https://i40.semanticinteroperability.org/rami/Documentation/index.html.

[97] Christoph Lange NP Irlan Grangel, "Semantic manufacturing ontology," 2018, https://i40.semanticinteroperability, https://i40.semantic-interoperability.org/ smo/. 
[98] D. M. R. Timothy Lebo and S. Sahoo, "PROV-O: the PROV ontology," 2013, https://www.w3.org, https://www.w3.org/ TR/prov-o/.

[99] S. T. Irlan Grangel, "SCORVoc vocabulary," 2015, https:// lucid-project.org/, https://github.com/vocol/scor.

[100] A. Gangemi, "The DOLCE+DnS ultralite ontology," 2010, http://ontologydesignpatterns.org/wiki/Ontology:

DOLCE+DnS_Ultralite. 